\title{
CONVERGENCE AND REGULARIZATION RESULTS FOR OPTIMAL CONTROL PROBLEMS WITH SPARSITY FUNCTIONAL
}

\author{
Gerd Wachsmuth ${ }^{1}$ And Daniel Wachsmuth ${ }^{2}$
}

\begin{abstract}
Optimization problems with convex but non-smooth cost functional subject to an elliptic partial differential equation are considered. The non-smoothness arises from a $L^{1}$-norm in the objective functional. The problem is regularized to permit the use of the semi-smooth Newton method. Error estimates with respect to the regularization parameter are provided. Moreover, finite element approximations are studied. A-priori as well as a-posteriori error estimates are developed and confirmed by numerical experiments.
\end{abstract}

Mathematics Subject Classification. 49M05, 65N15, 65N30, 49N45.

Received August 27, 2009. Revised February 9, 2010 and March 18, 2010.

Published online August 6, 2010.

\section{INTRODUCTION}

We investigate optimal control problems with a non-smooth objective functional of the following type: Minimize $J(y, u)$, which is given by

$$
J(y, u)=\frac{1}{2}\left\|y-y_{d}\right\|_{L^{2}(\Omega)}^{2}+\beta\|u\|_{L^{1}(\Omega)}+\frac{\alpha}{2}\|u\|_{L^{2}(\Omega)}^{2}
$$

subject to the elliptic equation

$$
\begin{aligned}
& A y=u \\
& \left.y\right|_{\Gamma}=0
\end{aligned}
$$

and to the control constraints

$$
u_{a}(x) \leq u(x) \leq u_{b}(x) \quad \text { a.e. on } \Omega .
$$

Here, $\Omega \subset \mathbb{R}^{n}, n=2,3$, is a bounded domain with boundary $\Gamma$. The operator $A$ is assumed to be a linear, elliptic second-order differential operator. The parameters $\alpha, \beta$ are non-negative parameters. Let us denote the optimal control problem (1.1)-(1.4) by (P). Such optimal control problems with $L^{1}$-functionals arise

\footnotetext{
Keywords and phrases. Non-smooth optimization, sparsity, regularization error estimates, finite elements, discretization error estimates.

1 Chemnitz University of Technology, Faculty of Mathematics, 09107 Chemnitz, Germany.

2 Johann Radon Institute for Computational and Applied Mathematics (RICAM), Austrian Academy of Sciences, Altenbergerstrae 69, 4040 Linz, Austria. daniel.wachsmuth@ricam.oeaw.ac.at
} 
if one tries to find the best location of the control actuator, see e.g. Stadler [27]. This is due to the following special property of the solutions: on sets, where the adjoint state is small, the optimal control must be zero. Consequently, the optimal control has small support, which gives an indication to choose the actuator location. Analogous observations can be made for optimization problems in sequence spaces involving $l^{1}$-norms, see the comments below.

The optimal control problem under consideration admits a unique optimal control that will be denoted by $u_{\alpha}$. For $\alpha=0$, the resulting optimization problem is convex but non-smooth, whereas for $\alpha>0$ the optimization problem admits a semi-smooth necessary optimality system, in this case, the parameter $\alpha$ acts as regularization and smoothing parameter. We are especially interested in the behavior of solutions for fixed $\beta \geq 0$ and $\alpha \rightarrow 0$. For related estimates in the case $\beta=0$ and with additional state constraints $y \leq y_{c}$, we refer to the recent work by Lorenz and Rösch [22].

In this work, we investigate two types of approximations for Problem $(\mathbf{P})$. First, we will study convergence of solutions if the regularization parameter $\alpha$ tends to zero. We prove that the $L^{2}$-norm of the regularization error of the control obeys

$$
\left\|u_{0}-u_{\alpha}\right\|_{L^{2}}=O\left(\alpha^{1 / 2}\right)
$$

see below Theorem 3.7. This is a novel result in the context of optimal control problems with inequality constraints. Secondly, we study finite-element approximations for the regularized problem, which yields approximations $u_{\alpha, h}$ of $u_{\alpha}$ in a finite-dimensional space. We prove the a-priori estimate

$$
\left\|u_{\alpha, h}-u_{\alpha}\right\|_{L^{2}}=O(h)
$$

which coincide with available results for smooth functionals, i.e. for $\beta=0$, see below Propositions 4.5 and 4.6 . Both a-priori results are combined in Section 5 to choose the regularization parameter $\alpha$ in dependence of the mesh size $h$ to obtain optimal convergence of $u_{0}-u_{\alpha, h}$. Moreover, localized a-posteriori error estimators of the type

$$
\left\|u_{\alpha, h}-u_{\alpha}\right\|_{L^{2}}^{2} \leq c \sum_{T \in \mathcal{T}_{h}} \eta_{T}^{2}
$$

are considered, where the error indicators $\eta_{T}$ can be used in an adaptive process to compute approximations of solutions $u_{\alpha}$ efficiently.

Let us comment on known results on a-priori and a-posteriori analysis of control constrained optimal control problems with $\alpha>0, \beta=0$. Basic a-priori estimates were derived by Falk [9], which yield that the $L^{2}$-error of the control is controlled by the mesh size $h$ like $O(h)$. Convergence results for the approximation of controls by linear elements can be found in e.g. in the work of Casas and Mateos [4]. The recently introduced variational discretization concept by Hinze [14] gives the error estimate $\left\|u-u_{h}\right\|_{L^{2}}=O\left(h^{2}\right)$. The same convergence order can be achieved by means of a post-processing step, see Meyer and Rösch [23].

A-posteriori error estimators of residual type were studied for instance by Liu et al. [18-20], and Hintermüller et al. [13]. In addition, many papers are devoted to the so-called goal-oriented error estimators, for an outline of the underlying ideas see the survey of Becker and Rannacher [2].

Finally, let us report about existing literature in the context of inverse problems involving minimization problems in $l^{1}$. There, e.g., a possibly noisy signal should be reconstructed with as less non-zero coefficients of the solution as possible. That is, the support of the solution should be as small as possible, leading to a minimization with $l^{0}$-functionals. In certain situations, the minimizer of $l^{1}$-functionals coincide with the minimizer of the $l^{0}$-problem, see Donoho [7], which justifies the use of $l^{1}$-functionals to compute the sparsest solution. Solution methods for the arising non-smooth problems are studied for instance by Daubechies et al. [6], Griesse and Lorenz [11], Jin et al. [16], Ramlau and Teschke [25]. Regularization error estimates under suitable source conditions for $(\alpha, \beta) \rightarrow(0,0)$ can be found for instance in Grasmair et al. [10] and Lorenz [21].

Optimization problems in $L^{1}$ and $l^{1}$ share some major properties: the resulting problems are convex and non-smooth. Furthermore, the optimality conditions imply that their solutions have potentially small support. The fundamental differences arise from the different underlying functional analytic structure: The space $l^{1}$ is 
the dual of the Banach space $c_{0}$, which yields that the unit ball $l^{1}$ is weak-star compact and that the $l^{1}$-norm is weak-star lower semicontinuous. This can be used to prove existence of solutions for optimization problems involving $l^{1}$-norms. The same argument does not apply for $L^{1}(\Omega)$ : This space is not the dual space of any normed linear space, hence the notation 'weak-star' makes no sense. Moreover, bounded sets in $L^{1}(\Omega)$ are in general not relatively weak compact due to the Dunford-Pettis theorem. In particular, the optimization problem (1.1)-(1.3) for $\alpha=0$ without additional constraints has no solution in $L^{1}(\Omega)$ in general. Here one has to resort to measures, see e.g. Clason and Kunisch [5]. Hence, the control constraints (1.4) are indispensable to prove existence of solutions of $(\mathbf{P})$.

For inverse problems, the question of convergence of solutions for $(\alpha, \beta) \rightarrow(0,0)$ is studied intensively. There, one is interested to obtain in the limit the solution of an operator equation. Compared to optimal control problems this corresponds to the case that the optimal state $y_{0}$ for $\alpha=0$ fulfills $y_{0}=y_{d}$, i.e. that the desired state can be reached. Due to the presence of the inequality constraints and due to $\beta \nrightarrow 0$ this cannot be expected in general. One main ingredient in the existing convergence proofs, are the so-called source conditions, where one assumes that $u_{0}$ lies in the range of a certain adjoint operator $\mathcal{S}^{*}$. In our case, this would mean that $u_{0}$ is in the range of the solution operator of an elliptic partial differential equation, which implies the regularity $u_{0} \in H^{1}(\Omega)$. This is not practical for problems with control constraints, since for $\alpha=0$ the optimal control $u_{0}$ is discontinuous in general with jumps along curves, which means that $u_{0} \notin H^{1}(\Omega)$. This makes the fulfillment of a range condition unlikely. In the proof of our convergence result, we rather used a structural assumption on the active sets, see below Theorem 3.7. For a more detailed comparison, we refer to the discussion in Section 3.2 below.

\section{Notations and assumptions}

Let $\Omega \subset \mathbb{R}^{d}, d=2,3$, be a bounded domain with Lipschitz boundary $\Gamma$. The operator $A$ is a uniformly elliptic differential operator defined by

$$
(A y)(x)=-\sum_{i, j=1}^{N} \frac{\partial}{\partial x_{i}}\left(a_{i j}(x) \frac{\partial}{\partial x_{j}} y(x)\right)+c_{0}(x) y(x)
$$

with functions $a_{i j} \in C^{0,1}(\bar{\Omega}), c_{0} \in L^{\infty}(\Omega)$, satisfying the condition $a_{i j}(x)=a_{j i}(x)$ and for some $\delta_{0}, \delta_{1}>0$

$$
\begin{aligned}
\delta_{0}\|y\|_{H^{1}(\Omega)}^{2} & \leq\langle A y, y\rangle_{H^{-1}, H^{1}} & & \forall y \in H_{0}^{1}(\Omega), \\
\left\langle A y_{1}, y_{2}\right\rangle_{H^{-1}, H^{1}} & \leq \delta_{1}\left\|y_{1}\right\|_{H^{1}(\Omega)}\left\|y_{2}\right\|_{H^{1}(\Omega)} & & \forall y_{1}, y_{2} \in H_{0}^{1}(\Omega) .
\end{aligned}
$$

Let us denote by $a(\cdot, \cdot)$ the bilinear form induced by $A$

$$
a(u, v)=\langle A u, v\rangle_{H^{-1}, H^{1}} .
$$

The elliptic equation is solved in the weak sense, i.e. the weak solution $y$ satisfies

$$
a(y, v)=(u, v) \quad \forall v \in H_{0}^{1}(\Omega) .
$$

The corresponding solution mapping is denoted by $\mathcal{S}$, which is a continuous linear injective operator from $H^{-1}(\Omega)$ to $H_{0}^{1}(\Omega)$. Thanks to the assumptions on the differential operator $A$ above, the operator $\mathcal{S}$ as well as its adjoint operator $\mathcal{S}^{\star}$ is continuous from $L^{2}(\Omega)$ to $L^{\infty}(\Omega)$, see e.g. [28].

Furthermore, functions $y_{d} \in L^{2}(\Omega), u_{a}, u_{b} \in L^{\infty}(\Omega) \cap H^{1}(\Omega), u_{a}(x) \leq 0 \leq u_{b}(x)$ a.e. on $\Omega$, are given. Please note, that the assumption $u_{a} \leq 0 \leq u_{b}$ is not a restriction. If one has, e.g., $u_{a}>0$ on a subset $\Omega_{1} \subset \Omega$, we can decompose the $L^{1}$-norm as $\|u\|_{L^{1}(\Omega)}=\|u\|_{L^{1}\left(\Omega \backslash \Omega_{1}\right)}+\int_{\Omega_{1}} u$. Hence, on $\Omega_{1}$ the $L^{1}$-norm in $U_{a d}$ is in fact a linear functional, and thus the problem can be handled in an analogous way. 


\section{EXISTENCE OF SOLUTIONS AND OPTIMALITY CONDITIONS}

In this section we prove existence and uniqueness of solutions. Moreover, we derive optimality conditions. In [27] this is done already for the case $\alpha>0$, but we will also handle the case $\alpha=0$.

Lemma 2.1. The problem (P) has a unique solution even in the cases $\alpha=0$ or $\beta=0$.

Proof. Since the solution mapping $\mathcal{S}$ is injective, it is easy to see that the reduced objective $\hat{J}(u):=J(\mathcal{S} u, u)$ is strictly convex and continuous. Furthermore, the set $U_{a d}$ is convex and weakly compact in $L^{2}(\Omega)$. Therefore, the existence and uniqueness of the optimal control follows from standard arguments [29].

Let us remark that it is also possible to prove the existence and uniqueness of the solution for $\alpha=0$ in a pure $L^{1}$ setting. That is, if we assume only $u_{a}, u_{b} \in L^{1}(\Omega)$ we have to state the problem in $L^{1}(\Omega)$ since $U_{a d} \not \subset L^{2}(\Omega)$. Therefore, we need higher regularity assumptions of the domain $\Omega$ to solve the elliptic equation with a right-hand side in $L^{1}(\Omega)$. Caused by the fact that $L^{1}(\Omega)$ is not reflexive, we can not prove the weak compactness of $U_{a d}$ by its boundedness. However, weak compactness can be proven directly, which gives the existence and uniqueness of a solution in $L^{1}(\Omega)$, see [30], p. 8.

Since the objective function is not smooth but convex with respect to $u$, we can use the calculus of subdifferentials, see e.g. [15], Chapter 0.3.2. The subdifferential of the $L^{1}(\Omega)$-norm is given by

$$
v \in \partial\|u\|_{L^{1}} \Leftrightarrow v(x)\left\{\begin{array}{ll}
=1 & u(x)>0 \\
\in[-1,1] & u(x)=0 \\
=-1 & u(x)<0
\end{array} \quad \text { for almost all } x \in \Omega, \quad v \in L^{\infty}(\Omega) .\right.
$$

Now we can characterize the solution of $(\mathbf{P})$ by a variational inequality, which is necessary and sufficient for the optimality of $u_{\alpha}$.

Lemma 2.2. The functions $u_{\alpha} \in U_{a d}$ and $y_{\alpha}=\mathcal{S} u_{\alpha}$ are the optimal solution of $(\mathbf{P})$ if and only if $u_{\alpha}$, the adjoint state $p_{\alpha}=\mathcal{S}^{\star}\left(y_{d}-y_{\alpha}\right)$ and a subgradient $\lambda_{\alpha} \in \beta \partial\left\|u_{\alpha}\right\|_{L^{1}}$ satisfy the variational inequality

$$
\left(-p_{\alpha}+\alpha u_{\alpha}+\lambda_{\alpha}, u-u_{\alpha}\right) \geq 0 \text { for all } u \in U_{a d} .
$$

Proof. Following [15] we can compute the necessary and sufficient optimality condition for the convex problem $\min _{u \in U_{a d}} \hat{J}(u)$ as follows: $u_{\alpha}$ is a solution if there exists $\lambda_{\alpha}^{\star} \in \partial \hat{J}\left(u_{\alpha}\right)$ such that for every $u \in U_{a d}$

$$
\left(\lambda_{\alpha}^{\star}, u-u_{\alpha}\right) \geq 0
$$

holds. We derive the subdifferential as

$$
\partial \hat{J}\left(u_{\alpha}\right)=-p_{\alpha}+\alpha u_{\alpha}+\beta \partial\left\|u_{\alpha}\right\|_{L^{1}},
$$

and so the variational inequality directly follows.

As in [29], p. 57, and [27], p. 4, one can discuss the variational inequality pointwise and get a pointwise relation of $u_{\alpha}$ and $p_{\alpha}$ as displayed in Figure 1. We see that $\left|p_{\alpha}\right|<\beta$ implies $u_{\alpha}=0$, which promotes the sparsity property of $u_{\alpha}$. See [27] for a more detailed discussion.

\section{Estimates OF THE REGUlARIZATION ERROR}

As already mentioned, one can compute solutions of $(\mathbf{P})$ with a semi-smooth Newton method in the case $\alpha>0$, where the method converge locally super-linearly, see [27], Theorem 4.3. This however does not hold for $\alpha=0$. Hence, it is natural to approximate the solution $u_{0}$ for $\alpha=0$ with the solutions $u_{\alpha}$ for $\alpha>0$. 


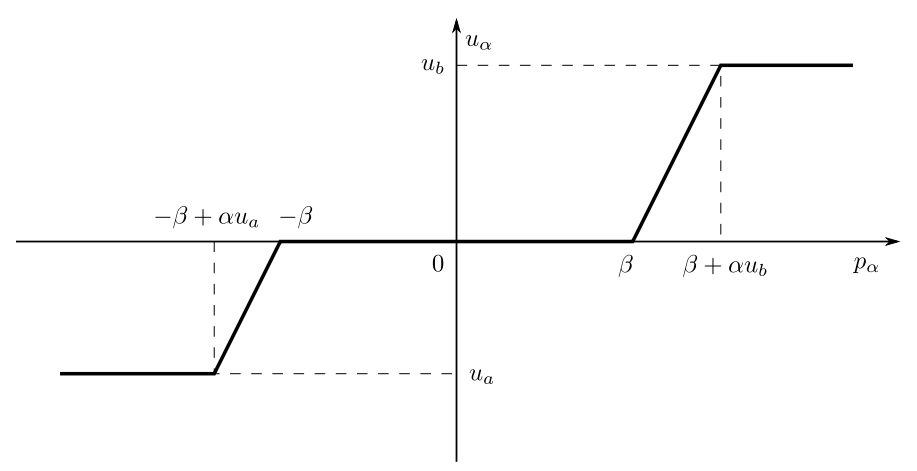

FiguRE 1. Relationship between $u_{\alpha}$ and $p_{\alpha}$.

\subsection{Estimation by regularity of the active sets}

At first, we derive an inequality that will be the starting point to obtain error estimates for the states and adjoints.

Lemma 3.1. The inequality

$$
\left\|y_{\alpha^{\prime}}-y_{\alpha}\right\|_{L^{2}}^{2}+\alpha\left\|u_{\alpha^{\prime}}-u_{\alpha}\right\|_{L^{2}}^{2} \leq\left(\alpha^{\prime}-\alpha\right)\left(u_{\alpha^{\prime}}, u_{\alpha}-u_{\alpha^{\prime}}\right)
$$

holds for all $\alpha>0, \alpha^{\prime} \geq 0$.

Proof. The solutions $u_{\alpha^{\prime}}, u_{\alpha}$ fulfill the variational inequalities

$$
\begin{aligned}
& \left(-p_{\alpha^{\prime}}+\alpha^{\prime} u_{\alpha^{\prime}}+\lambda_{\alpha^{\prime}}, v_{1}-u_{\alpha^{\prime}}\right) \geq 0 \\
& \left(-p_{\alpha}+\alpha u_{\alpha}+\lambda_{\alpha}, v_{2}-u_{\alpha}\right) \geq 0
\end{aligned}
$$

each for all admissible $v_{1}, v_{2} \in U_{a d}$. Testing with $v_{1}=u_{\alpha}$ and $v_{2}=u_{\alpha^{\prime}}$, and adding the inequalities leads to

$$
\left(p_{\alpha^{\prime}}-p_{\alpha}-\alpha^{\prime} u_{\alpha^{\prime}}+\alpha u_{\alpha}-\lambda_{\alpha^{\prime}}+\lambda_{\alpha}, u_{\alpha^{\prime}}-u_{\alpha}\right) \geq 0 .
$$

Since $\lambda_{\alpha^{\prime}}$ and $\lambda_{\alpha}$ are subgradients of $\|\cdot\|_{L^{1}}$, we obtain

$$
\left(-\lambda_{\alpha^{\prime}}+\lambda_{\alpha}, u_{\alpha^{\prime}}-u_{\alpha}\right) \leq 0
$$

by using the monotonicity of the subdifferential. This gives

$$
\left(p_{\alpha^{\prime}}-p_{\alpha}-\alpha^{\prime} u_{\alpha^{\prime}}+\alpha u_{\alpha}, u_{\alpha^{\prime}}-u_{\alpha}\right) \geq 0
$$

which directly leads to

$$
\begin{aligned}
0 & \leq\left(\mathcal{S}^{\star}\left(y_{\alpha}-y_{\alpha^{\prime}}\right), u_{\alpha^{\prime}}-u_{\alpha}\right)-\alpha\left(u_{\alpha^{\prime}}-u_{\alpha}, u_{\alpha^{\prime}}-u_{\alpha}\right)+\left(\alpha-\alpha^{\prime}\right)\left(u_{\alpha^{\prime}}, u_{\alpha^{\prime}}-u_{\alpha}\right) \\
& =-\left\|y_{\alpha^{\prime}}-y_{\alpha}\right\|_{L^{2}}^{2}-\alpha\left\|u_{\alpha^{\prime}}-u_{\alpha}\right\|_{L^{2}}^{2}+\left(\alpha^{\prime}-\alpha\right)\left(u_{\alpha^{\prime}}, u_{\alpha}-u_{\alpha^{\prime}}\right) .
\end{aligned}
$$

This entails our claim.

Using $\alpha^{\prime}=0$ in the previous lemma we obtain the estimate

$$
\left\|y_{0}-y_{\alpha}\right\|_{L^{2}}^{2}+\alpha\left\|u_{0}-u_{\alpha}\right\|_{L^{2}}^{2} \leq \alpha\left(u_{0}, u_{0}-u_{\alpha}\right) .
$$


TABle 1. Partition of $\Omega$, used in Proof of Lemma 3.3.

\begin{tabular}{l|c|c|c}
\hline & $p_{0}<-\beta$ & $\left|p_{0}\right|<\beta$ & $p_{0}>\beta$ \\
\hline$p_{\alpha} \leq-\beta+\alpha u_{a}$ & $u_{0}=u_{\alpha}=u_{a}$ & $I_{1}$ & $I_{1}$ \\
\hline$p_{\alpha} \in\left(-\beta+\alpha u_{a},-\beta\right]$ & $I_{2}$ & $I_{1}$ & $I_{1}$ \\
\hline$\left|p_{\alpha}\right|<\beta$ & $I_{1}$ & $u_{0}=u_{\alpha}=0$ & $I_{1}$ \\
\hline$p_{\alpha} \in\left[\beta, \beta+\alpha u_{b}\right)$ & $I_{1}$ & $I_{1}$ & $I_{3}$ \\
\hline$p_{\alpha} \geq \beta+\alpha u_{b}$ & $I_{1}$ & $I_{1}$ & $u_{0}=u_{\alpha}=u_{b}$ \\
\hline
\end{tabular}

Since the admissible set is bounded due to the control constraints, we can conclude a first convergence result for the states and adjoints.

Corollary 3.2. The estimate

$$
\left\|y_{0}-y_{\alpha}\right\|_{L^{2}} \leq C \alpha^{1 / 2}, \quad\left\|p_{0}-p_{\alpha}\right\|_{L^{\infty}} \leq C \alpha^{1 / 2}
$$

holds for all $\alpha>0$.

Proof. The operator $\mathcal{S}^{\star}$ maps right-hand sides in $L^{2}(\Omega)$ to adjoints in $L^{\infty}(\Omega)$, which yields with $(3.1)$

$$
\left\|p_{0}-p_{\alpha}\right\|_{L^{\infty}}^{2} \leq \alpha\left\|\mathcal{S}^{\star}\right\|_{2 \rightarrow \infty}^{2}\left(u_{0}, u_{0}-u_{\alpha}\right)
$$

Since the scalar product $\left(u_{0}, u_{0}-u_{\alpha}\right)$ is bounded due to the control constraints, we find directly the stated convergence rates.

We will now show convergence rates for the error in the adjoint states imply convergence rates for the error in the controls. To this end, we have to make an assumption on the boundary of the set $\left\{\left|p_{0}\right|=\beta\right\}$. Analogous assumptions on the boundary of active sets can be found in connection with finite element error estimates for elliptic optimal control problems, see $[4,23]$.

Lemma 3.3. Let us assume that there exists a constant $C_{p}>0$ such that for every $\varepsilon \geq 0$ the estimate for the Lebesgue measure $\mu$ of $\left\{|| p_{0}|-\beta| \leq \varepsilon\right\}$ is bounded as:

$$
\mu\left(\left\{|| p_{0}|-\beta| \leq \varepsilon\right\}\right) \leq C_{p} \varepsilon
$$

Then we have for all $d \in(0,1]$

$$
\begin{aligned}
& \left\|p_{0}-p_{\alpha}\right\|_{L^{\infty}} \leq C \alpha^{d} \Rightarrow \quad\left\|u_{0}-u_{\alpha}\right\|_{L^{2}} \leq C^{\prime} \alpha^{d / 2} \\
& \text { and }\left\|p_{0}-p_{\alpha}\right\|_{L^{\infty}} \leq C^{\prime \prime} \alpha^{\frac{d+2}{4}} \text {. }
\end{aligned}
$$

Proof. Let us divide $\Omega$ in disjoint sets depending on the values of $p_{0}$ and $p_{\alpha}$, see also Table 1 ,

$$
\begin{aligned}
& I_{1}:=\left\{x \in \Omega: \beta \text { or }-\beta \text { lies between } p_{0} \text { and } p_{\alpha}\right\} \\
& I_{2}:=\left\{x \in \Omega: p_{0}, p_{\alpha} \leq-\beta \text { and } p_{\alpha} \geq-\beta+\alpha u_{a}\right\} \\
& I_{3}:=\left\{x \in \Omega: p_{0}, p_{\alpha} \geq+\beta \text { and } p_{\alpha} \leq \beta+\alpha u_{b}\right\} .
\end{aligned}
$$

Note that we can ignore the set $\left\{\left|p_{0}\right|=\beta\right\}$, since it has measure zero by assumption (3.3). Let us define the union $U=I_{1} \cup I_{2} \cup I_{3}$. On $\Omega \backslash U$ we have $u_{0}=u_{\alpha}$, while we can bound the measures of the sets $I_{1}, I_{2}$ and $I_{3}$. On $I_{1}$ the assumption ensures ||$p_{0}|-\beta| \leq\left|p_{0}-p_{\alpha}\right| \leq C \alpha^{d}$. On $I_{2}$ we have ||$p_{0}|-\beta|=\left|p_{0}+\beta\right| \leq \alpha u_{a}+C \alpha^{d}$ and on $I_{3}$ we have analogously ||$p_{0}|-\beta| \leq \alpha u_{b}+C \alpha^{d}$. So on the union $U$ we have ||$p_{0}|-\beta| \leq C_{b} \alpha+C \alpha^{d}$ with the constant $C_{b}=\max \left(\left\|u_{a}\right\|_{L^{\infty}},\left\|u_{b}\right\|_{L^{\infty}}\right)$ depending on the bounds $u_{a}, u_{b}$. Using $d \leq 1$ and $\alpha \leq 1$ we have 
||$p_{0}|-\beta| \leq\left(C+C_{b}\right) \alpha^{d}$. Now we can bound the measure $M=\mu(U)$ of this set and get $M \leq C_{p}\left(C+C_{b}\right) \alpha^{d}$. Due to the $L^{\infty}$ control constraints and $u_{0}=u_{\alpha}$ on $\Omega \backslash U$, we have by (3.1)

$$
\left\|u_{0}-u_{\alpha}\right\|_{L^{2}}^{2} \leq M\left\|u_{0}\right\|_{L^{\infty}}\left\|u_{0}-u_{\alpha}\right\|_{L^{\infty}} \leq 2 C_{p}\left(C+C_{b}\right) C_{b}^{2} \alpha^{d} .
$$

By (3.2) we obtain

$$
\left\|p_{0}-p_{\alpha}\right\|_{L^{\infty}} \leq C_{S}\left(C_{b}^{\prime}\right)^{1 / 2}\left(2 C_{p}\left(C+C_{b}\right) C_{b}^{2}\right)^{1 / 4} \alpha^{\frac{d+2}{4}},
$$

with the constants $C_{b}^{\prime}=\max _{u \in U_{a d}}\|u\|_{L^{2}}=\left\|\max \left(u_{b},-u_{a}\right)\right\|_{L^{2}}$ and $C_{S}=\left\|\mathcal{S}^{\star}\right\|_{2 \rightarrow \infty}$. Let us define $C^{\prime}:=$ $C_{b}\left(2 C_{p}\left(C+C_{b}\right)\right)^{1 / 2}$. Then we obtain the desired implication

$$
\left\|p_{0}-p_{\alpha}\right\|_{L^{\infty}} \leq C \alpha^{d} \Rightarrow \begin{aligned}
\left\|u_{0}-u_{\alpha}\right\|_{L^{2}} & \leq C^{\prime} \alpha^{d / 2} \\
\text { and }\left\|p_{0}-p_{\alpha}\right\|_{L^{\infty}} & \leq C_{S}\left(C_{b}^{\prime} C^{\prime}\right)^{1 / 2} \alpha^{\frac{d+2}{4}}
\end{aligned}
$$

for all $d \in(0,1]$.

With this lemma, we can prove a first convergence result.

Corollary 3.4. Under the assumptions of Lemma 3.3, there is for each $d<1 / 3$ a constant $C_{d}>0$ such that

$$
\left\|u_{0}-u_{\alpha}\right\|_{L^{2}} \leq C_{d} \alpha^{d}
$$

holds.

Proof. By Lemma 3.3, $\left\|p_{0}-p_{\alpha}\right\|_{L^{\infty}} \leq C \alpha^{d}$ implies $\left\|u_{0}-u_{\alpha}\right\|_{L^{2}} \leq C^{\prime} \alpha^{d / 2}$ and $\left\|p_{0}-p_{\alpha}\right\|_{L^{\infty}} \leq C^{\prime \prime} \alpha^{(d+2) / 4}$. By Corollary 3.2, we know already $\left\|p_{0}-p_{\alpha}\right\|_{L^{\infty}} \leq C \alpha^{1 / 2}$. Now, let us consider the sequence $d_{0}=1 / 2$, $d_{k+1}=\left(d_{k}+2\right) / 4$, which corresponds to the convergence rates of the adjoint states. It is monotonically increasing and has the limit 2/3. So we get for all $d<2 / 3$ a constant $C_{d}$ with $\left\|p_{0}-p_{\alpha}\right\|_{L^{\infty}} \leq C_{d} \alpha^{d}$ and $\left\|u_{0}-u_{\alpha}\right\|_{L^{2}} \leq C_{d} \alpha^{d / 2}$. This proves the claim.

This corollary provides us with convergence rates for the controls up to order $1 / 3-\varepsilon$. However, we observed higher convergence rates in our numerical experiments. We will now prove higher rates using sensitivity information with respect to the parameter $\alpha$. Similar techniques are used for path-following algorithms, see e.g. [26] for an application to an optimal control problem with state constraints.

At first let us state the following differentiability result, which is proven in the appendix.

Lemma 3.5. The mappings $\alpha \mapsto u_{\alpha}, \alpha \mapsto y_{\alpha}$ and $\alpha \mapsto p_{\alpha}$ are Gâteaux-differentiable into $L^{2}(\Omega), L^{2}(\Omega)$ and $L^{\infty}(\Omega)$, respectively, at almost all $\alpha>0$. With help of the sets

$$
\begin{aligned}
& I_{1}=\left\{p_{\alpha} \in\left(\beta, \beta+\alpha u_{b}\right)\right\}, \\
& I_{2}=\left\{p_{\alpha} \in\left(-\beta+\alpha u_{a},-\beta\right)\right\}
\end{aligned}
$$

the derivatives are given as solutions of the system

$$
\begin{aligned}
\dot{u}_{\alpha} & =\frac{1}{\alpha^{2}}\left[\left(\beta-p_{\alpha}\right) \chi_{I_{1}}-\left(\beta+p_{\alpha}\right) \chi_{I_{2}}\right]+\frac{1}{\alpha} \dot{p}_{\alpha} \chi_{I_{1} \cup I_{2}} \\
\dot{y}_{\alpha} & =\mathcal{S} \dot{u}_{\alpha} \\
\dot{p}_{\alpha} & =-\mathcal{S}^{\star} \dot{y}_{\alpha} .
\end{aligned}
$$


Please note, that the claim of the lemma is only valid for almost all $\alpha>0$. To obtain differentiability for all $\alpha>0$, one has to resort to directional (Bouligand) derivatives. Then the resulting system for $\left(\dot{u}_{\alpha}, \dot{y}_{\alpha}, \dot{p}_{\alpha}\right)$ is not longer linear. However, the quantities $\left(\dot{u}_{\alpha}, \dot{y}_{\alpha}, \dot{p}_{\alpha}\right)$ can be interpreted as the solution of a suitably chosen inequality-constrained optimization problem. See [12] for further discussions and references.

Using the sensitivity information, we can estimate the distance from $y_{\alpha}$ to by an integral over the solutions of the sensitivity system.

Lemma 3.6. For all $\alpha>0$ we have the following estimate

$$
\left\|y_{0}-y_{\alpha}\right\|_{L^{2}} \leq \int_{0}^{\alpha}\left\|\dot{y}_{\tilde{\alpha}}\right\|_{L^{2}} \mathrm{~d} \tilde{\alpha} .
$$

Proof. Let us consider the function $f: \alpha \mapsto\left\|y_{0}-y_{\alpha}\right\|_{L^{2}}$. This function is Lipschitz continuous on each interval $[\varepsilon, M], \varepsilon>0$ by Lemma 3.1, and thus also absolutely continuous and almost everywhere differentiable. Moreover, it holds

$$
f\left(\alpha_{1}\right)-f\left(\alpha_{2}\right)=\int_{\alpha_{1}}^{\alpha_{2}} f^{\prime}(t) \mathrm{d} t
$$

for $\alpha_{i} \in[\varepsilon, M]$. Now, let us estimate the difference quotient

$$
\left|\frac{\left\|y_{0}-y_{\alpha+\delta}\right\|_{L^{2}}-\left\|y_{0}-y_{\alpha}\right\|_{L^{2}}}{\delta}\right| \leq\left\|\frac{1}{\delta}\left(y_{\alpha+\delta}-y_{\alpha}\right)\right\|_{L^{2}},
$$

and taking the limit $\delta \rightarrow 0$ yields

for almost all $\alpha>0$. Therefore we have

$$
\left|f^{\prime}(\alpha)\right| \leq\left\|\dot{y}_{\alpha}\right\|_{L^{2}}
$$

$$
f(\alpha)-f(\varepsilon) \leq \int_{\varepsilon}^{\alpha}\left\|\dot{y}_{\tilde{\alpha}}\right\|_{L^{2}} \mathrm{~d} \tilde{\alpha} \leq \int_{0}^{\alpha}\left\|\dot{y}_{\tilde{\alpha}}\right\|_{L^{2}} \mathrm{~d} \tilde{\alpha}
$$

for all $\varepsilon>0$, and passing to the limit $\varepsilon \rightarrow 0$ yields

$$
\left\|y_{0}-y_{\alpha}\right\| \leq \int_{0}^{\alpha}\left\|\dot{y}_{\tilde{\alpha}}\right\|_{L^{2}} \mathrm{~d} \tilde{\alpha}
$$

since $f$ is continuous in 0 with $f(0)=0$.

Now we can proof the main result of this section. The idea is to estimate the norm of the derivatives $\dot{y}_{\alpha}$, and then apply the previous lemma to obtain an estimate of the error.

Theorem 3.7. Let the regularity assumption

$$
\mu\left(\left\{|| p_{\alpha}|-\beta| \leq \varepsilon\right\}\right) \leq C_{p} \varepsilon
$$

be satisfied for $\alpha=0$. Then for each $d<1$ there is a constant $C_{d}$, such that

$$
\begin{aligned}
\left\|u_{0}-u_{\alpha}\right\|_{L^{2}} & \leq C_{d} \alpha^{d / 2}, \\
\left\|y_{0}-y_{\alpha}\right\|_{L^{2}} & \leq C_{d} \alpha^{d}, \\
\left\|p_{0}-p_{\alpha}\right\|_{L^{\infty}} & \leq C_{d} \alpha^{d}
\end{aligned}
$$

holds as $\alpha \rightarrow 0$.

If, in addition, the regularity condition (3.5) holds uniformly for almost all $\alpha \geq 0$, then there is a constant $C_{1}$ such that (3.6) is true for $d=1$. 
The first part of the theorem uses the same regularity condition as Lemma 3.3. Using the sensitivity information, we can however show a stronger result. If the regularity condition holds uniformly for $\alpha \geq 0$, then we can prove the full convergence rate. In this case the regularity condition implies that the set $\left\{|| p_{\alpha}|-\beta|=0\right\}$ has zero measure for $\alpha \geq 0$.

Proof. In this proof $C$ denotes a generic constant that is independent of $\alpha$. Testing (3.4b) with $\dot{y}_{\alpha}$ and (3.4c) with $\dot{u}_{\alpha}$ yields

$$
\left\|\dot{y}_{\alpha}\right\|_{L^{2}}^{2}=-\int_{\Omega} \dot{u}_{\alpha} \dot{p}_{\alpha} \mathrm{d} x
$$

Combining this result with equation (3.4a) we obtain with the notations $I_{1}$ and $I_{2}$ from Lemma 3.5

$$
\left\|\dot{y}_{\alpha}\right\|_{L^{2}}^{2}+\frac{1}{\alpha}\left\|\dot{p}_{\alpha} \chi_{I}\right\|_{L^{2}}^{2}=\frac{1}{\alpha^{2}}\left[\int_{I_{1}}\left(p_{\alpha}-\beta\right) \dot{p}_{\alpha} \mathrm{d} x+\int_{I_{2}}\left(p_{\alpha}+\beta\right) \dot{p}_{\alpha} \mathrm{d} x\right] .
$$

Then by definition of these sets, we have $p_{\alpha}-\beta \in\left(0, \alpha u_{b}\right)$ on $I_{1}$ and $p_{\alpha}+\beta \in\left(\alpha u_{a}, 0\right)$ on $I_{2}$. Let us define $I:=I_{1} \cup I_{2}$.

Together with $u_{a}, u_{b} \in L^{\infty}(\Omega)$ we can estimate the right hand side of (3.7) and obtain

$$
\left\|\dot{y}_{\alpha}\right\|_{L^{2}}^{2}+\frac{1}{\alpha}\left\|\dot{p}_{\alpha} \chi_{I}\right\|_{L^{2}}^{2} \leq \frac{C}{\alpha}\left\|\dot{p}_{\alpha} \chi_{I}\right\|_{L^{2}}\left\|\chi_{I}\right\|_{L^{2}}
$$

which implies

$$
\left\|\dot{y}_{\alpha}\right\|_{L^{2}} \leq \frac{C}{\sqrt{\alpha}}\left\|\chi_{I}\right\|_{L^{2}}
$$

Now, it remains to bound $\left\|\chi_{I}\right\|_{L^{2}}=\sqrt{\mu(I)}$.

Case 1. Inequality (3.5) holds for $\alpha=0$ and for almost all $\alpha>0$ :

The regularity assumption (3.5) yields

and with (3.8) we get

$$
\mu(I) \leq C \alpha
$$$$
\left\|\dot{y}_{\alpha}\right\|_{L^{2}} \leq C
$$

Now integration (Lem. 3.6) yields

$$
\left\|y_{0}-y_{\alpha}\right\|_{L^{2}} \leq C \alpha
$$

and we conclude with the smoothing property of $\mathcal{S}^{\star}$ and Lemma 3.3

$$
\begin{aligned}
\left\|p_{0}-p_{\alpha}\right\|_{L^{\infty}} & \leq C \alpha \\
\left\|u_{0}-u_{\alpha}\right\|_{L^{2}} & \leq C \alpha^{1 / 2}
\end{aligned}
$$

Case 2. Inequality (3.5) holds for $\alpha=0$ :

In this case we use a bootstrapping argument similar to Corollary 3.4. Let us define $C_{b}:=\max \left(\left\|u_{a}\right\|_{\infty},\left\|u_{b}\right\|_{\infty}\right)$. Then we have

$$
I \subset\left\{|| p_{\alpha}|-\beta| \leq C_{b} \alpha\right\}
$$

Now, suppose that $\left\|p_{0}-p_{\alpha}\right\|_{\infty} \leq C \alpha^{d}$ with $d<1$ holds. Using ||$p_{0}|-\beta| \leq|| p_{\alpha}|-\beta|+\left|p_{\alpha}-p_{0}\right|$ we have for $\alpha<1$

$$
I \subset\left\{|| p_{\alpha}|-\beta| \leq C_{b} \alpha\right\} \subset\left\{|| p_{0}|-\beta| \leq C \alpha^{d}\right\} .
$$

Utilizing assumption (3.5), we conclude $\mu(I) \leq C \alpha^{d}$. Following the same steps as in Case 1, we obtain $\left\|\dot{y}_{\alpha}\right\|_{L^{2}} \leq C \alpha^{(d-1) / 2},\left\|y_{0}-y_{\alpha}\right\|_{L^{2}} \leq C \alpha^{(d+1) / 2}$ and $\left\|p_{0}-p_{\alpha}\right\|_{\infty} \leq C \alpha^{(d+1) / 2}$. Thus, we proved the implication

$$
\left\|p_{0}-p_{\alpha}\right\|_{\infty} \leq C \alpha^{d} \Rightarrow\left\|p_{0}-p_{\alpha}\right\|_{\infty} \leq C \alpha^{(d+1) / 2}
$$


Starting with $d=1 / 2$ (Cor. 3.2) and observing that the sequence $a_{0}=1 / 2, a_{n+1}=\left(a_{n}+1\right) / 2$ converges towards 1 ends the proof.

\subsection{Discussion of the regularity assumption and source conditions}

We proved the convergence results under the regularity assumption (3.3), which reads

$$
\mu\left(\left\{|| p_{0}|-\beta| \leq \varepsilon\right\}\right) \leq C_{p} \varepsilon .
$$

This assumption implies, that the set $\left\{|| p_{0}|-\beta|=0\right\}$ has zero measure. Consequently, the optimal control $u_{0}$ satisfies $u_{0}(x) \in\left\{u_{a}(x), 0, u_{b}(x)\right\}$ almost everywhere. This is typically observed for solutions of optimal control problems for $\alpha=0$. There the optimal control has discontinuities on the set $\left\{|| p_{0}|-\beta|=0\right\}$, which means that in general $u_{0} \notin H^{1}(\Omega)$ holds.

Let us comment on available source conditions for our optimal control problem. The considerations will be based on the inequality (3.1), i.e.

$$
\left\|y_{0}-y_{\alpha}\right\|_{L^{2}}^{2}+\alpha\left\|u_{0}-u_{\alpha}\right\|_{L^{2}}^{2} \leq \alpha\left(u_{0}, u_{0}-u_{\alpha}\right) .
$$

Following Lorenz and Rösch [22], let us assume that the following source condition is fulfilled: There exists $w \in L^{2}(\Omega)$ such that

Due to the properties of the projection, we have

$$
u_{0}=P_{U_{a d}}\left(\mathcal{S}^{*} w\right)
$$

$$
\left(u_{0}-\mathcal{S}^{*} w, u_{\alpha}-u_{0}\right) \geq 0 .
$$

Then (3.9) becomes

$$
\left\|y_{0}-y_{\alpha}\right\|_{L^{2}}^{2}+\alpha\left\|u_{0}-u_{\alpha}\right\|_{L^{2}}^{2} \leq \alpha\left(u_{0}, u_{0}-u_{\alpha}\right) \leq \alpha\left(\mathcal{S}^{*} w, u_{0}-u_{\alpha}\right)=\alpha\left(w, \mathcal{S}\left(u_{0}-u_{\alpha}\right)\right)=\alpha\left(w, y_{0}-y_{\alpha}\right),
$$

which gives

$$
\left\|y_{0}-y_{\alpha}\right\|_{L^{2}} \leq \alpha\|w\|_{L^{2}}, \quad\left\|u_{0}-u_{\alpha}\right\|_{L^{2}} \leq \sqrt{\alpha}\|w\|_{L^{2}} .
$$

However, the assumption on $u_{0}$ implies the regularity $u_{0} \in H^{1}(\Omega)$ for bounds $u_{a}, u_{b} \in H^{1}(\Omega)$, and is thus not compatible with the observation that $u_{0}$ is typically not in $H^{1}(\Omega)$. Other source conditions as developed in $[10,21]$ are not directly applicable, since they are tailored to the case $(\alpha, \beta) \rightarrow(0,0)$, while we are considering the convergence for $\alpha \rightarrow 0$ and fixed $\beta$.

\section{A Priori finite Element error analysis, $\alpha>0$}

As indicated, the optimal control problem with $\alpha>0$ is better suited for numerical computations. After studying the regularization error, we will now turn to the finite element analysis of the regularized problems.

Let us fix the assumptions on the discretization of Problem (P) by finite elements. First let us specify the notation of regular meshes. Each mesh $\mathcal{T}$ consists of closed cells $T$ (for example triangles, tetrahedra, etc.) such that $\bar{\Omega}=\bigcup_{T \in \mathcal{T}} T$ holds, which implies in particular that cells with edges/faces lying on the boundary are curved for smooth, non-polygonal $\Omega$. We assume that the mesh is regular in the following sense: For different cells $T_{i}, T_{j} \in \mathcal{T}, i \neq j$, the intersection $T_{i} \cap T_{j}$ is either empty or a node, an edge, or a face of both cells, i.e. hanging nodes are not allowed. Let us denote the size of each cell by $h_{T}=\operatorname{diam} T$ and define $h(\mathcal{T})=\max _{T \in \mathcal{T}} h_{T}$. For each $T \in \mathcal{T}$, we define $R_{T}$ to be the diameter of the largest ball contained in $T$.

We will work with a family of regular meshes $\mathcal{F}=\left\{\mathcal{T}_{h}\right\}_{h>0}$, where the meshes are indexed by their mesh size, i.e. $h\left(\mathcal{T}_{h}\right)=h$. We assume in addition that there exist two positive constants $\rho$ and $R$ such that

$$
\frac{h_{T}}{R_{T}} \leq R, \quad \frac{h}{h_{T}} \leq \rho
$$


hold for all cells $T \in \mathcal{T}_{h}$ and all $h>0$. With each mesh $\mathcal{T}_{h} \in \mathcal{F}$ we associate a finite-dimensional subspace $V_{h} \subset V$. For a given right-hand side $u$, we define $y_{h} \in V_{h}$ as the solution of the discrete weak formulation

$$
a\left(y_{h}, v_{h}\right)=\left(u, v_{h}\right) \quad \forall v_{h} \in V_{h},
$$

and we denote the corresponding solution operator by $\mathcal{S}_{h}$, i.e. $y_{h}=\mathcal{S}_{h} u$. In the following, we rely on an assumption on the spaces $V_{h}$, which is met by standard finite element choices.

Assumption 4.1. Let $u \in L^{2}(\Omega)$ be given. Let $y$ and $y_{h}$ be the solutions of (1.5) and (4.1), respectively. There exists a constant $c_{A}>0$ independent of $h, u$ such that

$$
\left\|y-y_{h}\right\|_{L^{2}}+h\left\|y-y_{h}\right\|_{H^{1}} \leq c_{A} h^{2}\|u\|_{L^{2}} .
$$

This assumption implies in particular $\left\|\mathcal{S}_{h}-\mathcal{S}\right\|_{L^{2} \rightarrow H^{1}} \leq c_{A} h$.

Now, let us introduce the control discretization. We will discretize the control utilizing positive basis functions. Here, we follow an approach introduced by Meyer et al. in [24]. Alternatively, one can follow the so-called variational approach of [14], in which one sets $U_{h}:=U$, see the corresponding arguments in Section 4.3.

Assumption 4.2. To each mesh we associate a finite-dimensional space $U_{h} \subset U$. There is a basis $\Phi_{h}=$ $\left\{\phi_{h}^{1}, \ldots, \phi_{h}^{N_{h}}\right\}$ of $U_{h}$, e.g. $U_{h}=\operatorname{span} \Phi_{h}$, where the basis functions $\phi_{h}^{i} \in L^{\infty}(\Omega)$ have the following properties:

$$
\phi_{h}^{i} \geq 0, \quad\left\|\phi_{h}^{i}\right\|_{\infty}=1 \quad \forall i=1 \ldots N_{h}, \quad \sum_{i=1}^{N_{h}} \phi_{h}^{i}(x)=1 \text { for a.a. } x \in \Omega .
$$

Furthermore, there are numbers $M, N$ such that following conditions are fulfilled for all $h$ and all $i=1 \ldots N_{h}$ : each support $\omega_{h^{i}}:=\operatorname{supp} \phi_{h^{i}}$ is connected, and it is contained in the union of at most $M$ adjacent cells $T \in \mathcal{T}_{h}$ sharing at least one vertex. Each cell $T \in \mathcal{T}_{h}$ is subset of at most $N$ supports $\omega_{h}^{i}$.

This assumption covers several commonly-used control discretizations, such as piecewise constant or linear functions, see [24]. Following the approach of [3,24], let us introduce a quasi-interpolation operator $\Pi_{h}: L^{1}(\Omega) \rightarrow$ $U_{h}$. The operator $\Pi_{h}$ is given by

$$
\Pi_{h}(u):=\sum_{i=1}^{N_{h}} \pi_{h}^{i}(u) \phi_{h}^{i} \text { with } \pi_{h}^{i}(u):=\frac{\int_{\Omega} u \phi_{h}^{i}}{\int_{\Omega} \phi_{h}^{i}} .
$$

Please note, that $\Pi_{h}$ is not a projection with respect to the $L^{2}$-scalar product. Nevertheless, the following orthogonality relation holds for $u \in L^{2}(\Omega)$

$$
\int_{\Omega}\left(u-\pi_{h}^{i}(u)\right) \phi_{h}^{i}=0 .
$$

Based on the assumptions on the mesh and on the control discretization, we have the following interpolation estimates. For the proofs, we refer to [3,24].

Lemma 4.3. There is a constant $c_{I}$ independent of $h$ such that

$$
h\left\|u-\Pi_{h} u\right\|_{L^{2}(\Omega)}+\left\|u-\Pi_{h} u\right\|_{H^{-1}(\Omega)} \leq c_{I} h^{2}\|\nabla u\|_{L^{2}(\Omega)}
$$

is fulfilled for all $u \in H^{1}(\Omega)$.

It remains to describe the discrete admissible set $U_{a d, h}$. We use the quasi-interpolation operator $\Pi_{h}$ to define new bounds by

$$
u_{a, h}=\Pi_{h} u_{a}=\sum_{i} u_{a, h}^{i} \phi_{h}^{i}=\sum_{i} \pi_{h}^{i}\left(u_{a}\right) \phi_{h}^{i}, \quad u_{b, h}=\Pi_{h} u_{b}=\sum_{i} u_{b, h}^{i} \phi_{h}^{i}
$$


Let us set

$$
U_{a d, h}:=\left\{u \in U_{h}: u_{a, h} \leq u \leq u_{b, h} \quad \text { a.e. on } \Omega\right\} .
$$

Here it may happen, that $u_{a, h}$ or $u_{b, h}$ are no longer admissible, i.e. $u_{a, h} \notin U_{a d}$ or $u_{b, h} \notin U_{a d}$, which gives in the end a not admissible discretization $U_{a d, h} \not \subset U_{a d}$. For the special case of constant upper and lower bounds $u_{a}$ and $u_{b}$, it holds $U_{a d, h} \subset U_{a d}$. Nevertheless, the admissible set $U_{a d, h}$ can be written equivalently in the following way.

Lemma 4.4. Let $u_{a, h}, u_{b, h}, U_{a d, h}$ be defined as above. Then it holds

$$
U_{a d, h}=\left\{u=\sum_{i} u^{i} \phi_{h}^{i}, u_{a, h}^{i} \leq u^{i} \leq u_{b, h}^{i}\right\} .
$$

Proof. The first part ' $\subset$ ' of (4.4) follows directly from Assumption 4.2, which gives $u_{a, h}^{i} \leq u_{h}^{i} \leq u_{b, h}^{i}$. Summation of $u_{a, h}^{i} \phi_{h}^{i}(x) \leq u^{i} \phi_{h}^{i}(x) \leq u_{b, h}^{i} \phi_{h}^{i}(x)$ yields also the second inclusion ' $\supset$ '.

Thanks to this lemma, the constraint $u_{h} \in U_{a d, h}$ can be transformed in simple box constraints of the coefficients of $u_{h}$, which enables to use efficient solution techniques for the resulting optimization problem.

Let us now define the discrete optimal control problem as: Minimize $J\left(y_{h}, u_{h}\right)$ subject to $u_{h} \in U_{a d, h}$ and

$$
a\left(y_{h}, v_{h}\right)=\left(u, v_{h}\right) \quad \forall v_{h} \in V_{h} .
$$

This represents an optimization problem, which is uniquely solvable. Let us denote its solution by $\left(y_{\alpha, h}, u_{\alpha, h}\right)$ with associated adjoint state $p_{\alpha, h}$ and subgradient $\lambda_{\alpha, h} \in \partial\left\|u_{h}\right\|_{L^{1}}$. Analogously to the continuous problem, one obtains the variational inequality

$$
\left(\alpha u_{\alpha, h}-p_{\alpha, h}+\lambda_{\alpha, h}, u_{h}-u_{\alpha, h}\right) \geq 0 \quad \forall u_{h} \in U_{a d, h}
$$

as necessary and sufficient optimality condition, see Lemma 2.2 .

We will now derive error estimates in terms of the mesh size $h$. At first, we will derive upper bounds of $\left\|u_{\alpha}-u_{\alpha, h}\right\|_{L^{2}}$ and $\left\|y_{\alpha}-y_{\alpha, h}\right\|_{L^{2}}$. For different choices of $U_{h}$, we have to proceed differently, which amounts in a number of analogous error estimates. Now, let us start to derive the basic error bound with the help of the variational inequalities (2.2) and (4.5).

Here, it would be nice if we could use $u_{\alpha}$ as a test function in the variational inequality (4.5), which characterizes $u_{\alpha, h}$. However, in general the function $u_{\alpha}$ does not belong to $U_{a d, h}$ and cannot be utilized as test function. To overcome this difficulty, let us introduce an approximation $\tilde{u}_{h} \approx u_{\alpha}$ with $\tilde{u}_{h} \in U_{a d, h}$, which is suitable as test function in (4.5).

The same arguments apply to $u_{\alpha, h}$. Here one cannot expect $u_{\alpha, h} \in U_{a d}$ if for instance the control bounds are not constants. Thus, let us take a function $\tilde{u} \approx u_{\alpha, h}$ that is feasible for the continuous problem, i.e. $\tilde{u} \in U_{a d}$, and can be used as test function in the variational inequality (2.2).

Now let us use the test function $\tilde{u}$ in (2.2) and the test function $\tilde{u}_{h}$ in (4.5). Adding the resulting inequalities we obtain

$$
\begin{aligned}
& \alpha\left\|u_{\alpha}-u_{\alpha, h}\right\|_{L^{2}}^{2} \leq\left(\alpha u_{\alpha, h}-p_{\alpha, h}, \tilde{u}_{h}-u_{\alpha}\right)+\left(\alpha u_{\alpha}-p_{\alpha}, \tilde{u}-u_{\alpha, h}\right)-\left(p_{\alpha, h}-p_{\alpha}, u_{\alpha}-u_{\alpha, h}\right) \\
&+\beta\left(\|\tilde{u}\|_{L^{1}}-\left\|u_{\alpha, h}\right\|_{L^{1}}+\left\|\tilde{u}_{h}\right\|_{L^{1}}-\left\|u_{\alpha}\right\|_{L^{1}}\right) .
\end{aligned}
$$


Using standard argumentations, see e.g. [9], one finds

$$
\begin{aligned}
\alpha\left\|u_{\alpha}-u_{\alpha, h}\right\|_{L^{2}}^{2}+\left\|y_{\alpha}-y_{\alpha, h}\right\|_{L^{2}}^{2} \leq & \left(\alpha u_{\alpha}-p_{\alpha}, \tilde{u}-u_{\alpha, h}+\tilde{u}_{h}-u_{\alpha}\right)+\alpha\left(u_{\alpha, h}-u_{\alpha}, \tilde{u}_{h}-u_{\alpha}\right) \\
& -\left(y_{\alpha, h}-y_{\alpha},\left(\mathcal{S}_{h}-\mathcal{S}\right) \tilde{u}_{h}+\mathcal{S}\left(\tilde{u}_{h}-u_{\alpha}\right)\right) \\
& -\left(y_{\alpha}-y_{d},\left(\mathcal{S}_{h}-\mathcal{S}\right)\left(\tilde{u}_{h}-u_{\alpha, h}\right)\right) \\
& +\beta\left(\|\tilde{u}\|_{L^{1}}-\left\|u_{\alpha, h}\right\|_{L^{1}}+\left\|\tilde{u}_{h}\right\|_{L^{1}}-\left\|u_{\alpha}\right\|_{L^{1}}\right) .
\end{aligned}
$$

Here, we can apply Assumption 4.1 to estimate $\mathcal{S}_{h}-\mathcal{S}$, which gives

$$
\begin{aligned}
\frac{\alpha}{2}\left\|u_{\alpha}-u_{\alpha, h}\right\|_{L^{2}}^{2}+\frac{1}{2}\left\|y_{\alpha}-y_{\alpha, h}\right\|_{L^{2}}^{2} \leq & \left\|\alpha u_{\alpha}-p_{\alpha}\right\|_{H^{1}}\left(\left\|\tilde{u}-u_{\alpha, h}\right\|_{H^{-1}}+\left\|\tilde{u}_{h}-u_{\alpha}\right\|_{H^{-1}}\right)+\alpha\left\|_{\tilde{u}_{h}}-u_{\alpha}\right\|_{L^{2}}^{2} \\
& +c_{A}^{2} h^{4}\left\|\tilde{u}_{h}\right\|_{L^{2}}^{2}+\|\mathcal{S}\|_{\mathcal{L}\left(H^{-1}, L^{2}\right)}^{2}\left\|\tilde{u}_{h}-u_{\alpha}\right\|_{H^{-1}}^{2} \\
& +c_{A} h^{2}\left\|y_{\alpha}-y_{d}\right\|_{L^{2}}\left(\left\|\tilde{u}_{h}-u_{\alpha}\right\|_{L^{2}}+\left\|u_{\alpha}-u_{\alpha, h}\right\|_{L^{2}}\right) \\
& +\beta\left(\|\tilde{u}\|_{L^{1}}-\left\|u_{\alpha, h}\right\|_{L^{1}}+\left\|\tilde{u}_{h}\right\|_{L^{1}}-\left\|u_{\alpha}\right\|_{L^{1}}\right)
\end{aligned}
$$

Let us define $\tilde{u}_{h}=\Pi_{h} u_{\alpha}$, which implies $\tilde{u}_{h} \in U_{a d, h}$. Then we have by Lemma 4.3

$$
h\left\|\tilde{u}_{h}-u_{\alpha}\right\|_{L^{2}}+\left\|\tilde{u}_{h}-u_{\alpha}\right\|_{H^{-1}} \leq c_{I} h^{2}\left\|u_{\alpha}\right\|_{H^{1}} .
$$

Denoting by $u_{\alpha}^{+}$and $u_{\alpha}^{-}$the positive and negative parts of $u_{\alpha}$, we find

$$
\left\|\tilde{u}_{h}\right\|_{L^{1}}=\left\|\Pi_{h} u_{\alpha}^{+}+\Pi_{h} u_{\alpha}^{-}\right\|_{L^{1}} \leq\left\|\Pi_{h} u_{\alpha}^{+}\right\|_{L^{1}}+\left\|\Pi_{h} u_{\alpha}^{-}\right\|_{L^{1}}=\left\|u_{\alpha}^{+}\right\|_{L^{1}}+\left\|u_{\alpha}^{-}\right\|_{L^{1}}=\left\|u_{\alpha}\right\|_{L^{1}} .
$$

Thus, this choice of $\tilde{u}_{h}$ yields

$$
\begin{aligned}
\frac{\alpha}{2}\left\|u_{\alpha}-u_{\alpha, h}\right\|_{L^{2}}^{2}+\frac{1}{2} \| y_{\alpha}- & y_{\alpha, h}\left\|_{L^{2}}^{2} \leq\right\| \alpha u_{\alpha}-p_{\alpha} \|_{H^{1}}\left(\left\|\tilde{u}-u_{\alpha, h}\right\|_{H^{-1}}+c_{I} h^{2}\left\|u_{\alpha}\right\|_{H^{1}}\right) \\
& +c_{I}^{2} h^{2} \alpha\left\|u_{\alpha}\right\|_{H^{1}}^{2}+c_{A}^{2} h^{4}\left\|\tilde{u}_{h}\right\|_{L^{2}}^{2}+\|\mathcal{S}\|_{\mathcal{L}\left(H^{-1}, L^{2}\right)}^{2} c_{I}^{2} h^{4}\left\|u_{\alpha}\right\|_{H^{1}}^{2} \\
& \quad+c_{A} h^{2}\left\|y_{\alpha}-y_{d}\right\|_{L^{2}}\left(c_{I} h\left\|u_{\alpha}\right\|_{H^{1}}+\left\|u_{\alpha}-u_{\alpha, h}\right\|_{L^{2}}+\beta\left(\|\tilde{u}\|_{L^{1}}-\left\|u_{\alpha, h}\right\|_{L^{1}}\right) .\right.
\end{aligned}
$$

Let us recall that for $\alpha>0$ the optimal control $u_{\alpha}$ has the regularity $u_{\alpha} \in H^{1}(\Omega)$. However, its $H^{1}$-norm depends on $\alpha$ :

$$
\left\|u_{\alpha}\right\|_{H^{1}} \leq \frac{1}{\alpha}\left\|p_{\alpha}\right\|_{H^{1}}+\left\|u_{a}\right\|_{H^{1}}+\left\|u_{b}\right\|_{H^{1}}
$$

Due to the control constraints, the $H^{1}$-norm of $p_{\alpha}$ is bounded independently of $\alpha \geq 0$. The quantity $\| \alpha u_{\alpha}-$ $p_{\alpha} \|_{H^{1}}$ is also bounded independently of $\alpha$ : on sets, where it holds $\alpha u_{\alpha}-p_{\alpha} \neq 0$, the control constraint is active or $u_{\alpha}=0$. There, the expression $\left\|p_{\alpha}\right\|_{H^{1}}+\alpha\left(\left\|u_{a}\right\|_{H^{1}}+\left\|u_{b}\right\|_{H^{1}}\right)$ realizes an upper bound of $\left\|\alpha u_{\alpha}-p_{\alpha}\right\|_{H^{1}}$. Altogether, we can choose $M>0$ large enough and independent of $\alpha, h$ and $\alpha_{0}>0$, such that it holds for all $\alpha_{0} \geq \alpha \geq 0, h \geq 0$

$$
\left\|p_{\alpha}\right\|_{H^{1}}+\alpha\left(\left\|u_{a}\right\|_{H^{1}}+\left\|u_{b}\right\|_{H^{1}}\right)+\left\|y_{\alpha}-y_{d}\right\|_{L^{2}}+\sup _{u_{h} \in U_{a d, h}}\left\|u_{h}\right\|_{L^{2}} \leq M,
$$

which implies immediately $\left\|u_{\alpha}\right\|_{H^{1}} \leq M \alpha^{-1}$. The inequality (4.9) becomes

$$
\begin{aligned}
\frac{\alpha}{2}\left\|u_{\alpha}-u_{\alpha, h}\right\|_{L^{2}}^{2}+\frac{1}{2}\left\|y_{\alpha}-y_{\alpha, h}\right\|_{L^{2}}^{2} \leq & M\left(\left\|\tilde{u}-u_{\alpha, h}\right\|_{H^{-1}}+c_{I} M h^{2} \alpha^{-1}\right) \\
& +c_{I}^{2} M^{2} h^{2} \alpha^{-1}+c_{A} M^{2} h^{4}+\|\mathcal{S}\|_{\mathcal{L}\left(H^{-1}, L^{2}\right)}^{2} c_{I}^{2} M^{2} h^{4} \alpha^{-2} \\
& +c_{A} h^{2} M\left(c_{I} M h \alpha^{-1}+\left\|u_{\alpha}-u_{\alpha, h}\right\|_{L^{2}}\right)+\beta\left(\|\tilde{u}\|_{L^{1}}-\left\|u_{\alpha, h}\right\|_{L^{1}}\right) .
\end{aligned}
$$


We will now distinguish different cases of discretizations and control bounds. The methods of proof will differ in the choices of $\tilde{u} \approx u_{\alpha, h}$.

\subsection{General control discretization, constant upper and lower bounds}

For constant $u_{a}, u_{b}$, we have $u_{a, h}^{i}=u_{a}, u_{b, h}^{i}=u_{b}$, which results in $u_{a, h}=u_{a}$ and $u_{b, h}=u_{b}$. Hence it holds $U_{a d, h} \subset U_{a d}$, which allows for the choice $\tilde{u}=u_{\alpha, h}$. Then inequality (4.10) implies:

Proposition 4.5. Let $u_{a}, u_{b}$ be constant. Then for every $\alpha_{0}>0, h_{0}>0$ there is a constant $C>0$ such that for all $\alpha \leq \alpha_{0}, h \leq h_{0}$ it holds

where $C$ is independent of $\alpha, h$.

$$
\left\|u_{\alpha}-u_{\alpha, h}\right\|_{L^{2}} \leq C\left(h \alpha^{-1}+h^{2} \alpha^{-3 / 2}\right)
$$

Proof. With the choice $\tilde{u}=u_{\alpha, h}$, inequality (4.10) gives

$$
\begin{aligned}
\frac{\alpha}{2}\left\|u_{\alpha}-u_{\alpha, h}\right\|_{L^{2}}^{2} \leq & \left(c_{I} M^{2}+c_{I}^{2} M^{2}\right) h^{2} \alpha^{-1}+c_{A}^{2} M^{2} h^{4}+\|\mathcal{S}\|_{\mathcal{L}\left(H^{-1}, L^{2}\right)}^{2} c_{I}^{2} M^{2} h^{4} \alpha^{-2} \\
& +c_{A} c_{I} M^{2} h^{3} \alpha^{-1}+\frac{\alpha}{4}\left\|u_{\alpha}-u_{\alpha, h}\right\|_{L^{2}}^{2}+2 c_{A}^{2} M^{2} h^{4} \alpha^{-1},
\end{aligned}
$$

which yields with suitable chosen $C>0$ for $\alpha \leq \alpha_{0}, h \leq h_{0}$

$$
\left\|u_{\alpha}-u_{\alpha, h}\right\|_{L^{2}} \leq C\left(h \alpha^{-1}+h^{2} \alpha^{-3 / 2}\right) .
$$

\subsection{Piecewise constant control discretization, variable control bounds}

Here, we choose piecewise constant control functions, that is, we require $\phi_{h}^{i}(x) \in\{0,1\}$ everywhere on $\Omega$ for all $i, h$. Hence the supports of two different trial functions are disjoint. Let us remark that the arguments in the proof rely on the assumption $u_{a} \leq 0 \leq u_{b}$.

We choose $\tilde{u}_{h}=\Pi_{h} u_{\alpha}$ as in the previous subsection. We set $\tilde{u}$ as

$$
\tilde{u}=\sum_{i=1}^{N_{h}}\left(\sigma_{a}^{i} u_{a}+\sigma_{b}^{i} u_{b}\right) \phi_{h}^{i}
$$

with coefficients chosen as

$$
\sigma_{a}^{i}=\left\{\begin{array}{ll}
u_{\alpha, h}^{i} / u_{a, h}^{i} & \text { if } u_{\alpha, h}^{i}<0, \\
0 & \text { if } u_{\alpha, h}^{i} \geq 0,
\end{array} \quad \sigma_{b}^{i}= \begin{cases}0 & \text { if } u_{\alpha, h}^{i} \leq 0 \\
u_{\alpha, h}^{i} / u_{b, h}^{i} & \text { if } u_{\alpha, h}^{i}>0 .\end{cases}\right.
$$

This implies $\sigma_{a}^{i}, \sigma_{b}^{i} \in[0,1], \sigma_{a}^{i} \sigma_{b}^{i}=0$ and $\tilde{u} \in U_{a d}$. Moreover, it holds

$$
\tilde{u}-u_{\alpha, h}=\sum_{i=1}^{N_{h}}\left(\sigma_{a}^{i}\left(u_{a}-u_{a, h}^{i}\right)+\sigma_{b}^{i}\left(u_{b}-u_{b, h}^{i}\right)\right) \phi_{h}^{i} .
$$

Following [24], Lemmas 4.4 and 4.5, one finds

$$
h\left\|\tilde{u}-u_{\alpha, h}\right\|_{L^{2}}+\left\|\tilde{u}-u_{\alpha, h}\right\|_{H^{-1}} \leq c h^{2}\left(\left\|\nabla u_{a}\right\|_{L^{2}}+\left\|\nabla u_{b}\right\|_{L^{2}}\right) .
$$

It remains to investigate the $L^{1}$-norm of $\tilde{u}$. Here, we obtain

$$
\|\tilde{u}\|_{L^{1}}=\sum_{i=1}^{N_{h}}\left(\sigma_{a}^{i} \int_{\Omega}\left|u_{a}\right| \phi_{h}^{i}+\sigma_{b}^{i} \int_{\Omega}\left|u_{b}\right| \phi_{h}^{i}\right)=\sum_{i=1}^{N_{h}}\left(\sigma_{a}^{i}\left|u_{a, h}^{i}\right|+\sigma_{b}^{i}\left|u_{b, h}^{i}\right|\right)=\left\|u_{\alpha, h}\right\|_{L^{1}},
$$


where we used essentially that the supports of the $\phi_{h}^{i}$ 's are pairwise disjoint. Using now this results on $\tilde{u}$ and the results for $\tilde{u}_{h}$ above, we obtain from the inequality (4.10):

Proposition 4.6. Let $u_{a}, u_{b} \in H^{1}(\Omega)$ satisfy $u_{a} \leq 0 \leq u_{b}$. The control space is discretized by piecewise constant trial functions as above. Then for every $\alpha_{0}>0, h_{0}>0$ there is a constant $C>0$ such that for all $\alpha \leq \alpha_{0}, h \leq h_{0}$ it holds

$$
\left\|u_{\alpha}-u_{\alpha, h}\right\|_{L^{2}} \leq C\left(h \alpha^{-1}+h^{2} \alpha^{-3 / 2}\right)
$$

where $C$ is independent of $\alpha, h$.

\subsection{Variational control discretization}

The error estimate for the variational control discretization is a simple consequence of (4.6). Following [14], we set $U_{h}=U$, which gives $U_{a d, h}=U_{a d}$.

Corollary 4.7. Let $U_{h}=U$. Then for every $\alpha_{0}>0, h_{0}>0$ there is a constant $C>0$ such that for all $\alpha \leq \alpha_{0}, h \leq h_{0}$ the $L^{2}$-error of the controls satisfies

$$
\left\|u_{\alpha}-u_{\alpha, h}\right\|_{L^{2}} \leq C h^{2} \alpha^{-1}
$$

with $C$ independent of $h, \alpha$.

Proof. Due to $U_{a d}=U_{a d, h}$, we can choose $\tilde{u}_{h}=u_{\alpha}$ and $\tilde{u}=u_{\alpha, h}$ in (4.7), and we obtain

$$
\alpha\left\|u_{\alpha}-u_{\alpha, h}\right\|_{L^{2}}^{2}+\left\|y_{\alpha}-y_{\alpha, h}\right\|_{L^{2}}^{2} \leq-\left(y_{\alpha, h}-y_{\alpha},\left(\mathcal{S}_{h}-\mathcal{S}\right) u_{\alpha}\right)-\left(y_{\alpha}-y_{d},\left(\mathcal{S}_{h}-\mathcal{S}\right)\left(u_{\alpha}-u_{\alpha, h}\right)\right),
$$

which immediately yields in terms of the constants introduced above

$$
\alpha\left\|u_{\alpha}-u_{\alpha, h}\right\|_{L^{2}}^{2}+\left\|y_{\alpha}-y_{\alpha, h}\right\|_{L^{2}}^{2} \leq\left(c_{A} M h^{2}\right)^{2}+\left(c_{A} M h^{2}\right)^{2} \alpha^{-1} .
$$

\subsection{Discretization of the $L^{1}$-norm}

Up to now, we assumed that $\lambda_{\alpha, h}$ belongs to the subgradient of the $L^{1}$-norm at $u_{\alpha, h}$. This property can be maintained for piecewise constant control trial functions. In general, depending on the choice of $U_{h}, \lambda_{\alpha, h}$ will not belong to a finite-dimensional subspace. For example, if $U_{h}$ consists of piecewise linear functions over triangles, then $\lambda_{h}$ may have jumps along lines $u_{h}=0$ that are not grid lines. To overcome this difficulty, we introduce an approximation of the $L^{1}$-norm in the objective functional with the additional feature that its subdifferential can be represented by a finite-dimensional subspace. As it will turn out, this additional approximation step will not disturb the convergence estimate, in fact, both the error orders $h$ and $\alpha$ as well as the leading constant in the estimate remain unchanged.

Let us define now the approximation of the $L^{1}$-norm by

$$
\left\|u_{h}\right\|_{L^{1}, h}:=\sum_{i=1}^{N_{h}}\left|u_{h}^{i}\right| \int_{\Omega} \phi_{h}^{i}
$$

which is a weighted $l^{1}$-norm of the coefficients of $u_{h}$, thus it is a norm on $U_{h}$.

Let $u_{h}, v_{h} \in U_{h}$ be given with $\lambda_{h}^{i} \in \partial\left|u_{h}^{i}\right|, i=1 \ldots N_{h}$. Then we have by the construction of $\lambda_{h}^{i}$

$$
\sum_{i=1}^{N_{h}} \lambda_{h}^{i}\left(u_{h}^{i}-v_{h}^{i}\right) \int_{\Omega} \phi_{h}^{i} \leq \sum_{i=1}^{N_{h}}\left(\left|u_{h}^{i}\right|-\left|v_{h}^{i}\right|\right) \int_{\Omega} \phi_{h}^{i}=\left\|u_{h}\right\|_{L^{1}, h}-\left\|v_{h}\right\|_{L^{1}, h} .
$$


Here, we see that the subgradient of $\|\cdot\|_{L^{1}, h}$ can be represented by finitely many coefficients. Now, let us return to the a-priori error analysis. Let $u_{\alpha, h}$ be the solution of the discretized problem

$$
\min \frac{1}{2}\left\|y_{h}-y_{d}\right\|_{L^{2}}^{2}+\beta\left\|u_{h}\right\|_{L^{1}, h}+\frac{\alpha}{2}\left\|u_{h}\right\|_{L^{2}}^{2}
$$

subject to the discretized equation (4.1) and the control constraints $u_{h} \in U_{a d, h}$. Then there exists a discrete adjoint state $p_{\alpha, h}$ and numbers $\lambda_{\alpha, h}^{i} \in \partial\left|u_{h}^{i}\right|, i=1 \ldots N_{h}$. Using the variational inequalities, we obtain instead of (4.6) the slightly different estimate

$$
\begin{aligned}
& \alpha\left\|u_{\alpha}-u_{\alpha, h}\right\|_{L^{2}}^{2} \leq\left(\alpha u_{\alpha, h}-p_{\alpha, h}, \tilde{u}_{h}-u_{\alpha}\right)+\left(\alpha u_{\alpha}-p_{\alpha}, \tilde{u}-u_{\alpha, h}\right)-\left(p_{\alpha, h}-p_{\alpha}, u_{\alpha}-u_{\alpha, h}\right) \\
&+\beta\left(\|\tilde{u}\|_{L^{1}}-\left\|u_{\alpha, h}\right\|_{L^{1}, h}+\left\|\tilde{u}_{h}\right\|_{L^{1}, h}-\left\|u_{\alpha}\right\|_{L^{1}}\right),
\end{aligned}
$$

where the approximative $L^{1}$-norm instead of the $L^{1}$-norm is applied to $u_{\alpha, h}$ and $\tilde{u}_{h}$. We will now estimate the approximative $L^{1}$-norms against the $L^{1}$-norm. At first, we obtain

$$
\left\|u_{\alpha, h}\right\|_{L^{1}}=\int_{\Omega}\left|\sum_{i=1}^{N_{h}} u_{\alpha, h}^{i} \phi_{h}^{i}\right| \leq \int_{\Omega} \sum_{i=1}^{N_{h}}\left|u_{\alpha, h}^{i} \phi_{h}^{i}\right|=\sum_{i=1}^{N_{h}}\left|u_{\alpha, h}^{i}\right| \int_{\Omega} \phi_{h}^{i}=\left\|u_{\alpha, h}\right\|_{L^{1}, h} .
$$

Let us define $\tilde{u}_{h}=\Pi_{h} u_{\alpha}$ as above. Then we find

$$
\begin{aligned}
\left\|\tilde{u}_{h}\right\|_{L^{1}, h} & =\left\|\Pi_{h} u_{\alpha}^{+}+\Pi_{h} u_{\alpha}^{-}\right\|_{L^{1}, h} \leq\left\|\Pi_{h} u_{\alpha}^{+}\right\|_{L^{1}, h}+\left\|\Pi_{h} u_{\alpha}^{-}\right\|_{L^{1}, h} \\
& =\sum_{i=1}^{N_{h}}\left(\int_{\Omega} u_{\alpha}^{+} \phi_{h}^{i}-\int_{\Omega} u_{\alpha}^{-} \phi_{h}^{i}\right)=\sum_{i=1}^{N_{h}} \int_{\Omega}\left|u_{\alpha}\right| \phi_{h}^{i}=\left\|u_{\alpha}\right\|_{L^{1}} .
\end{aligned}
$$

That is, inequality (4.14) implies

$$
\begin{aligned}
\alpha\left\|u_{\alpha}-u_{\alpha, h}\right\|_{L^{2}}^{2} \leq & \left(\alpha u_{\alpha, h}-p_{\alpha, h}, \tilde{u}_{h}-u_{\alpha}\right)+\left(\alpha u_{\alpha}-p_{\alpha}, \tilde{u}-u_{\alpha, h}\right) \\
& -\left(p_{\alpha, h}-p_{\alpha}, u_{\alpha}-u_{\alpha, h}\right)+\beta\left(\|\tilde{u}\|_{L^{1}}-\left\|u_{\alpha, h}\right\|_{L^{1}}\right),
\end{aligned}
$$

compare also the inequality (4.6). Hence, we can proceed as above to obtain:

Corollary 4.8. Let $u_{\alpha, h}$ be the solution of the discrete problem (4.13) with approximated $L^{1}$-norm (4.12). Let the assumptions of Proposition 4.5 or Proposition 4.6 on $U_{h}, u_{a}, u_{b}$ be satisfied. Then for every $\alpha_{0}>0, h_{0}>0$ there is a constant $C>0$ such that for all $\alpha \leq \alpha_{0}, h \leq h_{0}$ the $L^{2}$-error of the controls satisfies

$$
\left\|u_{\alpha}-u_{\alpha, h}\right\|_{L^{2}} \leq C\left(h \alpha^{-1}+h^{2} \alpha^{-3 / 2}\right)
$$

with $C>0$ independent of $\alpha, h$, where the constant $C$ is the same as in Propositions 4.5 or 4.6.

Finally, we will give an interpretation of the coefficients $\lambda_{\alpha, h}^{i}$ as coefficients for certain trial functions. Let us construct a dual basis $\left\{\mu_{h}^{i}\right\}$ to $\left\{\phi_{h}^{i}\right\}$ satisfying

$$
\mu_{h}^{i} \in L^{\infty}(\Omega), \int_{\Omega} \mu_{h}^{i} \phi_{h}^{j}=\delta_{i, j} \quad \forall i, j=1 \ldots N_{h} .
$$

Let us denote by $\mathcal{M}_{h}=\left(m_{i j}\right), m_{i j}=\int_{\Omega} \phi_{h}^{i} \phi_{h}^{j}$, the mass matrix of the $\phi_{h}^{i}$ 's. This matrix is invertible with inverse matrix $\mathcal{M}_{h}^{-1}=\left(m^{i j}\right)$. Setting $\mu_{h}^{i}=\sum_{j=1}^{N_{h}} m^{i j} \phi_{h}^{j}$ yields a dual basis with the properties stated above. 
Defining $\lambda_{h}=\sum_{i=1}^{N_{h}} \lambda_{h}^{i}\left(\int_{\Omega} \phi_{h}^{i}\right) \mu_{h}^{i}$ with the coefficients $\lambda_{h}^{i}$ as above, we obtain

$$
\left(\lambda_{h}, u_{h}-v_{h}\right)=\sum_{i=1}^{N_{h}} \lambda_{h}^{i}\left(u_{h}^{i}-v_{h}^{i}\right)\left(\int_{\Omega} \phi_{h}^{i}\right) \leq\left\|u_{h}\right\|_{L^{1}, h}-\left\|v_{h}\right\|_{L^{1}, h}
$$

Therefore we have $\lambda_{h} \in \partial\left\|u_{h}\right\|_{L^{1}, h}$.

\subsection{Open problem: General control discretization, variable control bounds}

Let us remark, that it is an open problem to prove analogous error estimates for the general control discretization as introduced above in Assumption 4.2 if the control constraints are not constant. That means in particular, that for the discretization of controls by piecewise linear functions and non-trivial constraints no a-priori estimates can be proven. Here, it is open to construct an approximating function $\tilde{u} \approx u_{\alpha, h}$ with $\tilde{u} \in U_{a d}$ such that it holds

and

$$
h\left\|\tilde{u}-u_{\alpha, h}\right\|_{L^{2}}+\left\|\tilde{u}-u_{\alpha, h}\right\|_{H^{-1}} \leq c h^{2}
$$

$$
\|\tilde{u}\|_{L^{1}} \leq\left\|u_{\alpha, h}\right\|_{L^{1}}+c h^{2} .
$$

That is, a technique unifying the results of Sections 4.1 and 4.2 is missing.

\section{Simultaneous error estimates}

In this section we want to use the proven convergence results to obtain an error estimate for $\left\|u_{0}-u_{\alpha, h}\right\|_{L^{2}}$. If we use the triangle inequality, we have

$$
\left\|u_{0}-u_{\alpha, h}\right\|_{L^{2}} \leq\left\|u_{\alpha}+u_{\alpha, h}\right\|_{L^{2}}+\left\|u_{0}-u_{\alpha}\right\|_{L^{2}} \leq C\left(h \alpha^{-1}+h^{2} \alpha^{-3 / 2}+\alpha^{d}\right)
$$

with $d \leq 1 / 2$. Now we couple the mesh size $h$ to the regularization parameter $\alpha$. Since the error estimate is a polynomial in $h$ and $\alpha$ we suggest to use $h=\alpha^{\gamma}$ with $\gamma>0$. Now we have

$$
\left\|u_{0}-u_{\alpha}\right\|_{L^{2}} \leq C\left(\alpha^{\gamma-1}+\alpha^{2 \gamma-3 / 2}+\alpha^{d}\right),
$$

hence the order of convergence is $g=\min (\gamma-1,2 \gamma-3 / 2, d)$. In order to maintain convergence for $\alpha \rightarrow 0$, we require $g>0$, which implies $\gamma>1$ immediately. Since $\gamma-1<2 \gamma-3 / 2$ for all $\gamma>1$ we get $g=\min (\gamma-1, d)$. So the best possible order of convergence is $d$, and to reach this order we must have $\gamma-1 \geq d$. If we now choose $\gamma=3 / 2$ this will hold for all $d \leq 1 / 2$, which results in

$$
\left\|u_{0}-u_{\alpha, h}\right\|_{L^{2}} \leq C^{\prime} \alpha^{d} .
$$

Therefore the discretization does not influence the order of convergence if we choose meshes that are fine enough according to $h \sim \alpha^{3 / 2}$.

\section{A POSTERIORI ERROR ESTIMATOR}

Here, we will develop an a-posteriori error estimate from the difference of $u_{\alpha}$ and the computed approximation $u_{h}$ of the discrete solution $u_{\alpha, h}:\left\|u_{h}-u_{\alpha}\right\|_{L^{2}}$ for $\alpha>0$.

Let be given $y_{h}, p_{h} \in V_{h}, u_{h} \in U_{a d, h} \cap U_{a d}, \lambda_{h} \in L^{2}(\Omega)$. We will derive the error estimate without assuming that $\left(y_{h}, u_{h}, p_{h}, \lambda_{h}\right)$ are solutions of the discrete problem.

By optimality of $\left(y_{\alpha}, u_{\alpha}\right)$ we know

$$
0 \leq J\left(\mathcal{S} u_{h}, u_{h}\right)-J\left(y_{\alpha}, u_{\alpha}\right),
$$


which is equivalent to

$$
\begin{aligned}
0 \leq \frac{1}{2} & \left\|\mathcal{S} u_{h}-y_{d}\right\|_{L^{2}}^{2}-\frac{1}{2}\left\|y_{\alpha}-y_{d}\right\|_{L^{2}}+\frac{\alpha}{2}\left\|u_{h}\right\|_{L^{2}}^{2}-\frac{\alpha}{2}\left\|u_{\alpha}\right\|_{L^{2}}^{2} \\
& +\beta\left\|u_{h}\right\|_{L^{1}}-\beta\left\|u_{\alpha}\right\|_{L^{1}}+\left(A\left(\mathcal{S} u_{h}\right)-u_{h}-A y_{\alpha}+u_{\alpha}, p_{h}\right) \\
=- & \frac{1}{2}\left\|\mathcal{S} u_{h}-y_{\alpha}\right\|_{L^{2}}^{2}-\frac{\alpha}{2}\left\|u_{h}-u_{\alpha}\right\|_{L^{2}}^{2}+\left(\alpha u_{h}-p_{h}, u_{h}-u_{\alpha}\right) \\
& +\beta\left\|u_{h}\right\|_{L^{1}}-\beta\left\|u_{\alpha}\right\|_{L^{1}}+\left(A p_{h}+\mathcal{S} u_{h}-y_{d}, \mathcal{S} u_{h}-y_{\alpha}\right)
\end{aligned}
$$

since $A\left(\mathcal{S} u_{h}\right)=u_{h}, A y_{\alpha}=u_{\alpha}$, and $p_{h} \in H_{0}^{1}(\Omega)$ holds. Let us take $\tilde{\lambda} \in \partial \beta\left\|u_{h}\right\|_{L^{1}}$, which implies $\beta\left\|u_{h}\right\|_{L^{1}}-$ $\beta\left\|u_{\alpha}\right\|_{L^{1}} \leq\left(\tilde{\lambda}, u_{h}-u_{\alpha}\right)$. Furthermore, let us compute a function $\delta_{h}$, such that

$$
\left(\alpha u_{h}-p_{h}+\lambda_{h}+\delta_{h}, u-u_{h}\right) \geq 0 \quad \forall u \in U_{a d} .
$$

In fact, if $u_{h}, p_{h}, \lambda_{h}$ are known such a function $\delta_{h}$ can be computed, see e.g. [8,17]. We proceed with

$$
\begin{aligned}
0 \leq- & \frac{1}{2}\left\|\mathcal{S} u_{h}-y_{\alpha}\right\|_{L^{2}}^{2}-\frac{\alpha}{2}\left\|u_{h}-u_{\alpha}\right\|_{L^{2}}^{2}+\left(\alpha u_{h}-p_{h}+\lambda_{h}, u_{h}-u_{\alpha}\right) \\
& +\left(\tilde{\lambda}-\lambda_{h}, u_{h}-u_{\alpha}\right)+\left(A p_{h}+\mathcal{S} u_{h}-y_{d}, \mathcal{S} u_{h}-y_{\alpha}\right)
\end{aligned}
$$

which implies

$$
\begin{aligned}
0 \leq- & \frac{1}{2}\left\|\mathcal{S} u_{h}-y_{\alpha}\right\|_{L^{2}}^{2}-\frac{\alpha}{2}\left\|u_{h}-u_{\alpha}\right\|_{L^{2}}^{2}-\left(\delta_{h}, u_{h}-u_{\alpha}\right) \\
& +\inf _{\tilde{\lambda} \in \partial \beta\left\|u_{h}\right\|_{L^{1}}}\left(\tilde{\lambda}-\lambda_{h}, u_{h}-u_{\alpha}\right)+\left(A p_{h}+\mathcal{S} u_{h}-y_{d}, \mathcal{S} u_{h}-y_{\alpha}\right) .
\end{aligned}
$$

Denoting by $c_{L^{2}, H^{-1}}$ the norm of the embedding $L^{2}(\Omega) \rightarrow H^{-1}(\Omega)$, we obtain by the Cauchy-Schwarz inequality

$$
\begin{aligned}
\frac{1}{2}\left\|\mathcal{S} u_{h}-y_{\alpha}\right\|_{L^{2}}^{2}+\frac{\alpha}{4}\left\|u_{h}-u_{\alpha}\right\|_{L^{2}}^{2} & \leq \frac{4}{\alpha}\left\{\left\|\delta_{h}\right\|_{L^{2}}^{2}+\inf _{\tilde{\lambda} \in \partial \beta\left\|u_{h}\right\|_{L^{1}}}\left\|\tilde{\lambda}-\lambda_{h}\right\|_{L^{2}}^{2}\right. \\
& \left.+2\|\mathcal{S}\|_{L^{2} \rightarrow H_{0}^{1}}^{2}\left(\left\|A p_{h}+y_{h}-y_{d}\right\|_{H^{-1}}^{2}+c_{L^{2}, H^{-1}}^{2}\|\mathcal{S}\|_{H^{-1} \rightarrow L_{2}}^{2}\left\|A y_{h}-u_{h}\right\|_{H^{-1}}^{2}\right)\right\}
\end{aligned}
$$

Thus, we found an upper bound of the errors in control and state:

Theorem 6.1. Let be given $y_{h}, p_{h} \in V_{h}, u_{h} \in U_{a d, h} \cap U_{a d}, \lambda_{h} \in L^{2}(\Omega)$. Let $\left(y_{\alpha}, u_{\alpha}\right)$ be the solution of (P). Then it holds

$$
\begin{aligned}
\frac{1}{2}\left\|\mathcal{S} u_{h}-y_{\alpha}\right\|_{L^{2}}^{2}+\frac{\alpha}{4} \| u_{h}- & u_{\alpha} \|_{L^{2}}^{2} \leq \frac{4}{\alpha}\left\{\left\|\delta_{h}\right\|_{L^{2}}^{2}+\inf _{\tilde{\lambda} \in \partial \beta\left\|u_{h}\right\|_{L^{1}}}\left\|\tilde{\lambda}-\lambda_{h}\right\|_{L^{2}}^{2}\right. \\
& \left.+2\|\mathcal{S}\|_{L^{2} \rightarrow H_{0}^{1}}^{2}\left(\left\|A p_{h}+y_{h}-y_{d}\right\|_{H^{-1}}^{2}+c_{L^{2}, H^{-1}}^{2}\|\mathcal{S}\|_{H^{-1} \rightarrow L_{2}}^{2}\left\|A y_{h}-u_{h}\right\|_{H^{-1}}^{2}\right)\right\}
\end{aligned}
$$

Lower bounds of the error can be derived following the recent work of Li et al. [19] provided that $\left(y_{h}, u_{h}\right)$ is the solution of the discretized problem with associated adjoint state $p_{h}$ and subgradient $\lambda_{h}$.

To incorporate the estimate above in an adaptive refinement procedure it has to be specified how the error estimator can be evaluated cell-wise. The function $\delta_{h}$ can be computed as follows

$$
\delta_{h}(x)= \begin{cases}-\left(\alpha u_{h}-p_{h}+\lambda_{h}\right)^{-} & \text {if } u_{h}(x)=u_{a}(x) \\ -\left(\alpha u_{h}-p_{h}+\lambda_{h}\right) & \text { if } u_{a}(x)<u_{h}(x)<u_{b}(x) \\ -\left(\alpha u_{h}-p_{h}+\lambda_{h}\right)^{+} & \text {if } u_{h}(x)=u_{b}(x) .\end{cases}
$$


Although $\delta_{h}$ is not a grid function, it is a computationally easy task to evaluate $\left\|\delta_{h}\right\|_{L^{2}(T)}$ for each cell of the mesh. Similarly, a function $\tilde{\lambda}_{h} \in \partial \beta\left\|u_{h}\right\|_{L^{1}}$ can be constructed that realizes the minimum of $\left\|\tilde{\lambda}-\lambda_{h}\right\|_{L^{2}}$ :

$$
\tilde{\lambda}_{h}(x)=\beta \begin{cases}1 & \text { if } u_{h}(x)>0 \\ \max \left(-1, \min \left(\lambda_{h}(x), 1\right)\right) & \text { if } u_{h}(x)=0 \\ -1 & \text { if } u_{h}(x)<0\end{cases}
$$

The $H^{-1}$-residuals can be estimated by the standard residual estimate

$$
\left\|A y_{h}-u_{h}\right\|_{H^{-1}}^{2} \leq c_{1} \sum_{T \in \mathcal{T}_{h}}\left(h_{T}^{2}\left\|A y_{h}-u_{h}\right\|_{L^{2}(T)}^{2}+\sum_{E \subset \partial T \backslash \partial \Omega} h_{E}\left\|\left[\frac{\partial y_{h}}{\partial n_{A}}\right]\right\|_{L^{2}(E)}^{2}\right) .
$$

Here, the constant $c_{1}$ does depend on $\Omega$ and the shape regularity of $\left\{\mathcal{T}_{h}\right\}$. Altogether, we obtain the following computable error estimate.

Theorem 6.2. Let the assumptions of the previous Theorem 6.1 be satisfied. Let $\delta_{h}$ and $\tilde{\lambda}_{h}$ be computed according to (6.2) and (6.3), respectively. Then it holds

$$
\left\|\mathcal{S} u_{h}-y_{\alpha}\right\|_{L^{2}}^{2}+\alpha\left\|u_{h}-u_{\alpha}\right\|_{L^{2}}^{2} \leq \frac{c}{\alpha} \sum_{T \in \mathcal{T}_{h}} \eta_{T}^{2}
$$

with a constant $c$ depending on $\Omega$ and the shape regularity of $\left\{\mathcal{T}_{h}\right\}$ but independent of $\alpha$ and the actual mesh $\mathcal{T}_{h}$. The cell-wise quantities $\eta_{T}$ are defined by

$$
\begin{aligned}
\eta_{T}:=\left\|\delta_{h}\right\|_{L^{2}(T)}^{2}+\left\|\tilde{\lambda}_{h}-\lambda_{h}\right\|_{L^{2}(T)}^{2}+h_{T}^{2}\left(\left\|A y_{h}-u_{h}\right\|_{L^{2}(T)}^{2}+\left\|A p_{h}+y_{h}-y_{d}\right\|_{L^{2}(T)}^{2}\right) & \\
& +\sum_{E \subset \partial T \backslash \partial \Omega} h_{E}\left(\left\|\left[\frac{\partial y_{h}}{\partial n_{A}}\right]\right\|_{L^{2}(E)}^{2}+\left\|\left[\frac{\partial p_{h}}{\partial n_{A}}\right]\right\|_{L^{2}(E)}^{2}\right) .
\end{aligned}
$$

\section{NumeriCAL RESULTS}

\subsection{Constructed problems with known solutions}

In this section we show constructed problems of the type (1.1). For convenience we choose $A=-\Delta$. The first problem is a one-dimensional one, which is suitable to test the regularization errors estimates of Section 3 , since in our experience the discretization error dominates the regularization error for higher dimensional domains. 
Example 7.1. First we choose $\Omega=(0,1)$ for $\alpha=0, \beta=1 / 2$. Further we set $-u_{a}=u_{b}=30$. It can be calculated easily, that

$$
\begin{aligned}
& \bar{p}(x)=\sin (2 \pi x) \\
& \bar{u}(x)= \begin{cases}u_{a} & \text { where } x \in(1 / 12,5 / 12) \\
u_{b} & \text { where } x \in(7 / 12,11 / 12) \\
0 & \text { elsewhere }\end{cases} \\
& \bar{y}(x)=-30 \begin{cases}-x / 6 & \text { where } x \in[0,1 / 12) \\
x^{2} / 2-x / 4+1 / 288 & \text { where } x \in[1 / 12,5 / 12) \\
x / 6-1 / 12 & \text { where } x \in[5,7 / 12) \\
-x^{2} / 2+3 x / 4-73 / 288 & \text { where } x \in[7,11 / 12) \\
-x / 6+1 / 6 & \text { where } x \in[11,1]\end{cases} \\
& y_{d}=\bar{y}-\Delta \bar{p} \\
& =\bar{y}+4 \pi^{2} \sin (2 \pi x)
\end{aligned}
$$

satisfies the optimality condition (2.2) (compare Fig. 1).

Example 7.2. This is a problem with $\Omega=(0,2)^{2}, \alpha=0, \beta$ arbitrary, $u_{a}=-1, u_{b}=5$. For convenience we set $r=\left((1-x)^{2}+\left(1-y^{2}\right)\right)^{1 / 2}$. The rotationally symmetric functions

$$
\begin{gathered}
\bar{p}(r)=\beta \begin{cases}4 / 3-432 r^{4} & \text { where } r \in[0,1 / 6) \\
5 / 3-24 r^{2} & \text { where } r \in[1 / 6,1 / 3) \\
19 / 5-312 r^{2} / 5+864 r^{4} / 5 & \text { where } r \in[1 / 3,1 / 2) \\
-144 r^{4}+96 r^{2}-16 & \text { where } r \in[1 / 2,1 / \sqrt{3})\end{cases} \\
\Delta \bar{p}(r)=\beta \begin{cases}-6912 r^{2} & \text { where } r \in[0,1 / 6) \\
-96 & \text { where } r \in[1 / 6,1 / 3) \\
13824 r^{2} / 5-1248 / 5 & \text { where } r \in[1 / 3,1 / 2) \\
-2304 r^{2}+384 & \text { where } r \in[1 / 2,1 / \sqrt{3})\end{cases} \\
\bar{u}(r)=\left\{\begin{array}{lll}
u_{b} & \text { where } r \in[0,1 / 6) & \text { where } r \in[0,1 / 6) \\
u_{a} & \text { where } r \in[1 / 3,1 / 2) & \text { where } r \in[1 / 6,1 / 3)
\end{array}\right. \\
\bar{y}(r)= \begin{cases}5 r^{2} / 4+5 \log (1 / 2) / 72+\log (2 / 3) / 8 \\
5(\log (3 r)+1 / 2) / 72+\log (2 / 3) / 8 \\
-r^{2} / 4+\log (2 r) / 8+1 / 16 & \text { where } r \in[1 / 3,1 / 2)\end{cases} \\
y_{d}=\bar{y}-\Delta \bar{p}
\end{gathered}
$$

fulfill the optimality system. For convenience of the reader the plots of these functions can be found in Figures 2 and 3 . 

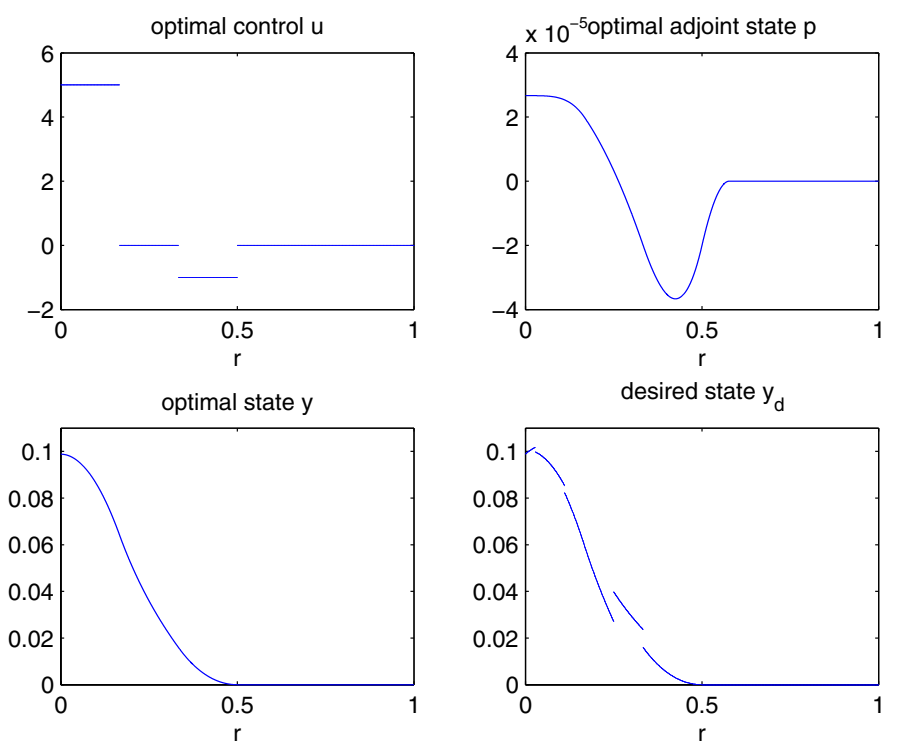

Figure 2. Cross section of Example 7.2, $\alpha=0, \beta=2 \times 10^{-5}$.

optimal control u

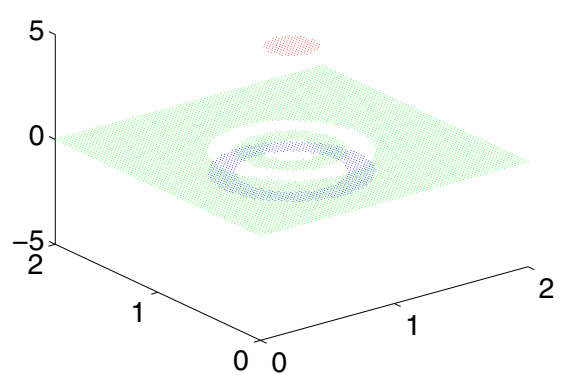

optimal state y

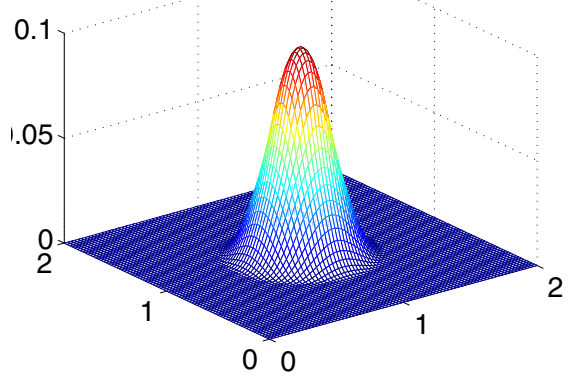

optimal adjoint state $\mathrm{p}$

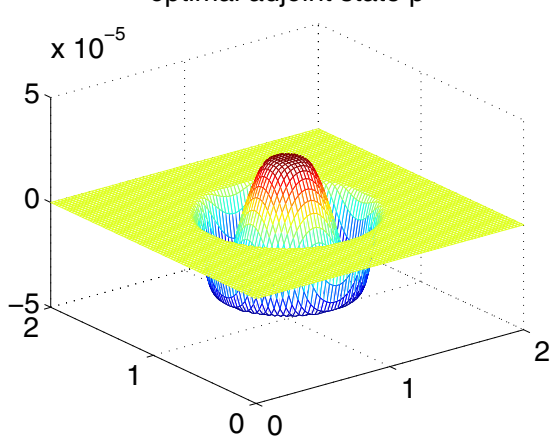

desired state $y_{d}$

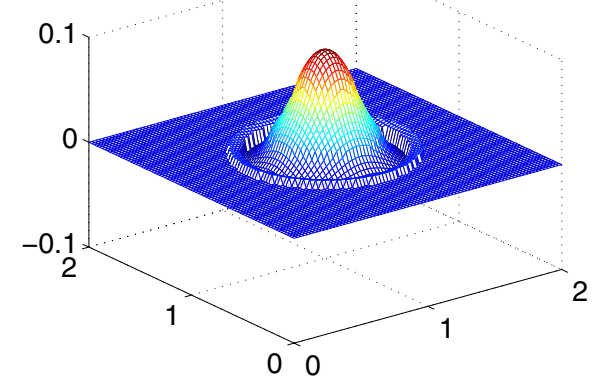

FiguRE 3. Example 7.2, $\alpha=0, \beta=2 \times 10^{-5}$. 
Example 7.3. This is a problem with $\Omega=(0,1)^{2}, 0<\alpha<\beta$ arbitrary, $u_{a}=-1, u_{b}=54 / 7$. For convenience we set $r=\left((1 / 2-x)^{2}+\left(1 / 2-y^{2}\right)\right)^{1 / 2}$. Then for

$$
\begin{array}{ll}
a=23328 \beta-5832 \alpha-5832 \alpha u_{b} & b=-9720 \beta+2268 \alpha+2592 \alpha u_{b} \\
c=1296 \beta-288 \alpha-378 \alpha u_{b} & d=-55 \beta+12 \alpha+18 \alpha u_{b} \\
e=-432 \beta+648 \alpha & f=108 \beta-216 \alpha
\end{array}
$$

the optimality system is fulfilled with

$$
\begin{aligned}
& \bar{p}(r)= \begin{cases}-162 \alpha u_{b} r^{2}+\beta+3 \alpha u_{b} / 2 & \text { where } r \in[0,1 / 18) \\
+\beta+\alpha \bar{u}(r) & \text { where } r \in[1 / 18,1 / 9) \\
a r^{3}+b r^{2}+c r+d & \text { where } r \in[1 / 9,1 / 6) \\
-\beta+\alpha \bar{u}(r) & \text { where } r \in[1 / 6,2 / 9) \\
324 \alpha(r-1 / 4)^{2}-\beta-5 \alpha / 4 & \text { where } r \in[2 / 9,5 / 18) \\
-\beta+\alpha \bar{u}(r) & \text { where } r \in[5 / 18,1 / 3) \\
e(r-1 / 3)^{3}+f(r-1 / 3)^{2}+18 \alpha(r-1 / 3)-\beta & \text { where } r \in[1 / 3,1 / 2)\end{cases} \\
& \Delta \bar{p}(r)= \begin{cases}-648 \alpha u_{b} & \text { where } r \in[0,1 / 18) \\
\alpha u_{b}-18 / r & \text { where } r \in[1 / 18,1 / 9) \\
9 a r+4 b+c / r & \text { where } r \in[1 / 9,1 / 6) \\
\alpha-18 / r & \text { where } r \in[1 / 6,2 / 9) \\
324 \alpha(4-1 /(2 r)) & \text { where } r \in[2 / 9,5 / 18) \\
18 \alpha / r & \text { where } r \in[5 / 18,1 / 3) \\
e(9 r-4+1 /(3 r))+f(4-2 /(3 r))+18 \alpha / r & \text { where } r \in[1 / 3,1 / 2)\end{cases} \\
& \bar{u}(r)= \begin{cases}u_{b} & \text { where } r \in[0,1 / 18) \\
-18 u_{b}(r-1 / 9) & \text { where } r \in[1 / 18,1 / 9) \\
0 & \text { where } r \in[1 / 9,1 / 6) \\
-18(x-1 / 6) & \text { where } r \in[1 / 6,2 / 9) \\
u_{a} & \text { where } r \in[2 / 9,5 / 18) \\
-6+18 r & \text { where } r \in[5 / 18,1 / 3)\end{cases} \\
& \bar{y}(r)= \begin{cases}u_{b} r^{2} / 4+\bar{y}(1 / 18)-1 / 168 & \text { where } r \in[0,1 / 18) \\
u_{b} r^{2} / 2-2 u_{b} r^{3}-\log (9 r) / 252+\bar{y}(1 / 9)-5 / 189 & \text { where } r \in[1 / 18,1 / 9) \\
\log (6 r) / 36+\bar{y}(1 / 6) & \text { where } r \in[1 / 9,1 / 6) \\
3 r^{2} / 4-2 r^{3}+\log (9 r / 2) / 72+\bar{y}(2 / 9)-11 / 729 & \text { where } r \in[1 / 6,2 / 9) \\
-r^{2} / 4+91 \log (18 r / 5) / 1944+25 / 1296+\bar{y}(5 / 18) & \text { where } r \in[2 / 9,5 / 18) \\
-3 r^{2} / 2+2 r^{3}+\log (3 r) / 9+5 / 54 & \text { where } r \in[5 / 18,1 / 3)\end{cases} \\
& y_{d}=\bar{y}-\Delta \bar{p} \text {. }
\end{aligned}
$$

These functions are plotted in Figures 4 and 5.

\subsection{Verification of regularization error}

In order to verify the estimates of the regularization error obtained in Section 3, we solved Example 7.1, because this one dimensional problem allows solving with very fine meshes. We choose the approximation parameter $\alpha>0$ in order to use a semi-smooth Newton method. The mesh parameters $h$ are set to $h=\frac{1}{10} \alpha^{3 / 2}$ 

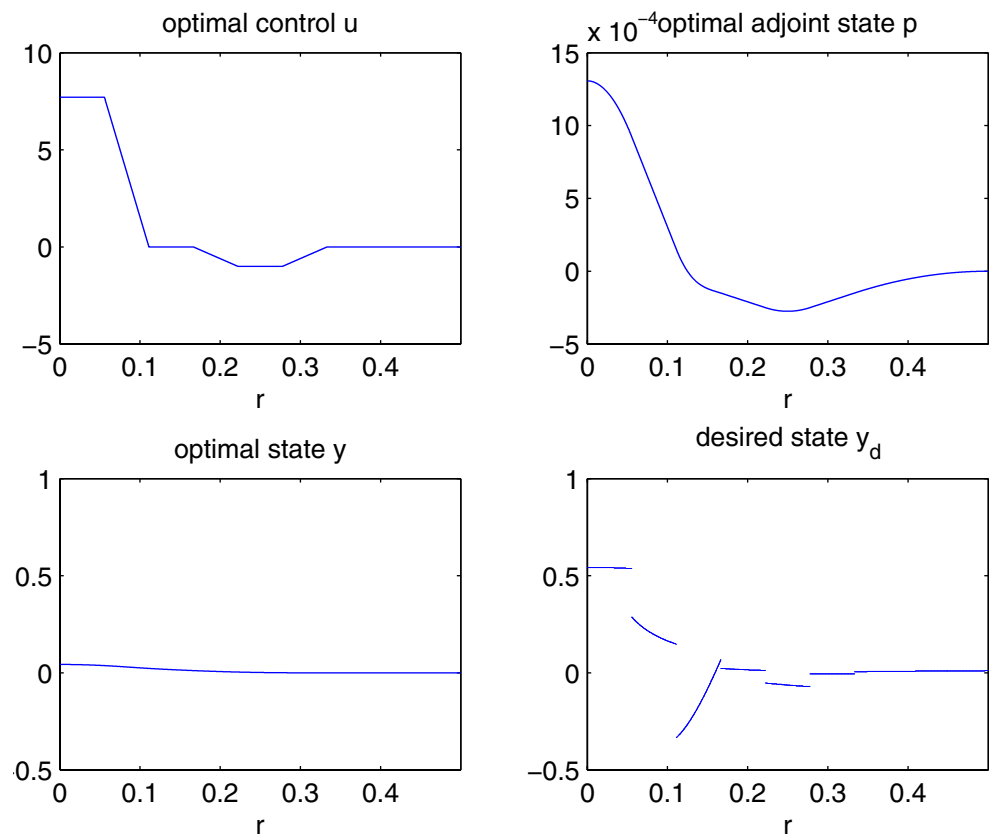

Figure 4. Cross section of Example 7.3, $\alpha=10^{-4}, \beta=1.5 \times 10^{-4}$.
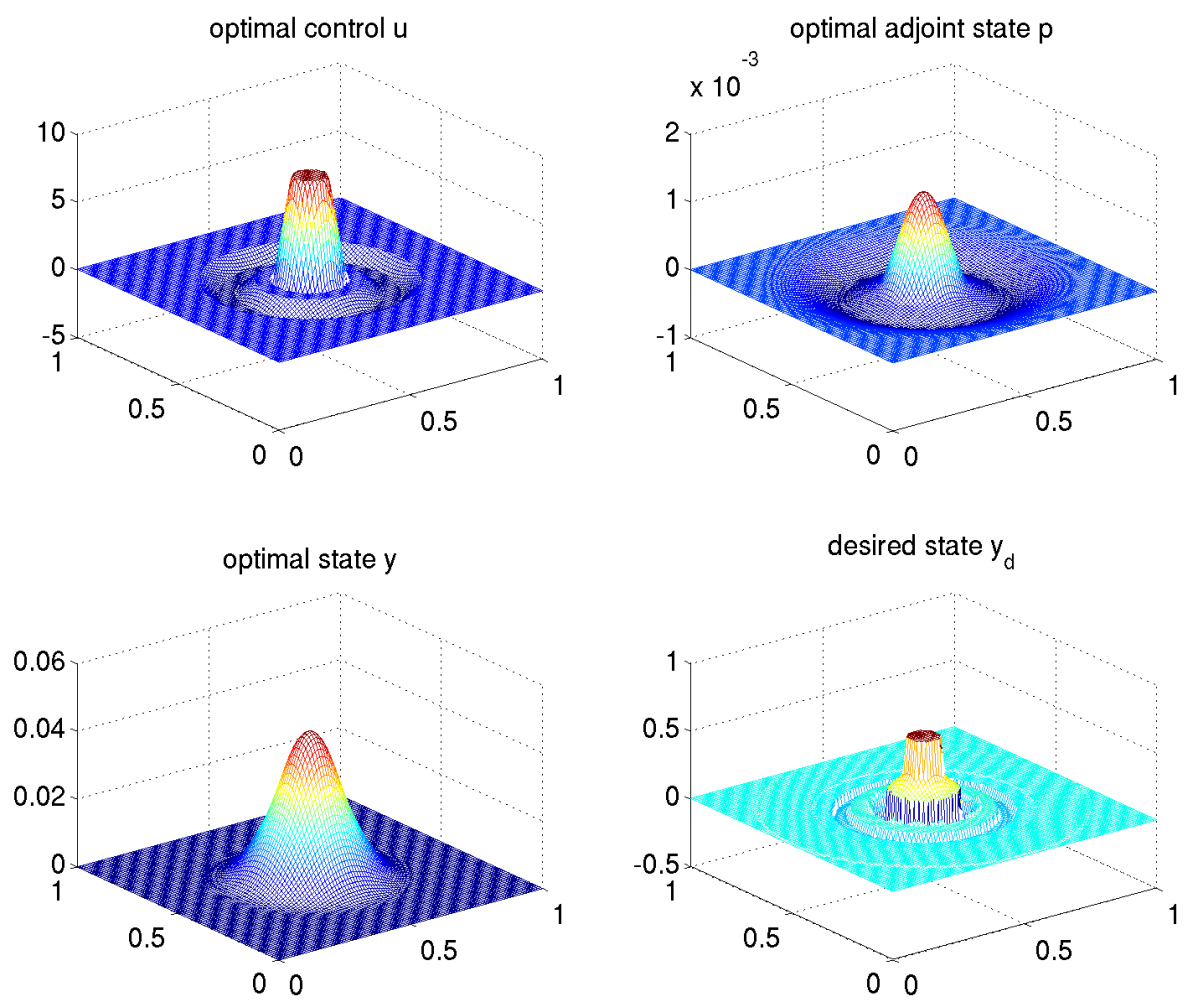

Figure 5. Example 7.3, $\alpha=10^{-4}, \beta=1.5 \times 10^{-4}$. 


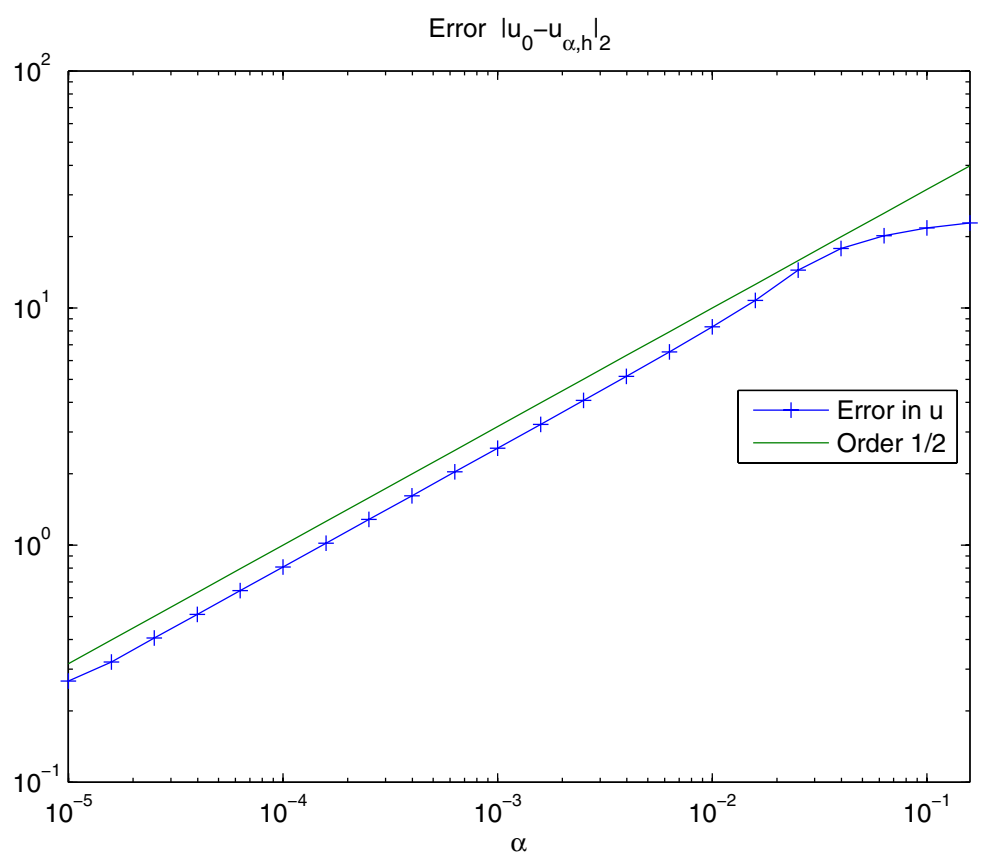

FiguRE 6. Solution of Example $7.1, h=0.1 \alpha^{3 / 2}$.

like recommended in Section 5. The unknowns were discretized with linear FEM trial functions. For the solution of the nonlinear system the semi-smooth Newton method by Stadler [27] was used. The error $\left\|u_{0}-u_{\alpha, h}\right\|_{L^{2}}$ is displayed in Figure 6. And we observed that the experimental order of convergence coincides with the theoretically obtained rate $1 / 2$.

\subsection{A-posteriori error estimates and adaptive mesh refinement}

We used the error estimator of Section 6 in a mesh adaptation procedure. Given a solution $\left(y_{\alpha, H}, u_{\alpha, H}, p_{\alpha, H}\right.$, $\left.\lambda_{\alpha, H}\right)$ on a coarse grid $\mathcal{T}_{H}$, a subset of triangles $\mathcal{T}_{H}^{\prime} \subset \mathcal{T}_{H}$ were marked for refinement. The subset was chosen such that

$$
\sum_{T \in \mathcal{T}_{H}^{\prime}} \eta_{T}^{2} \geq \theta^{2} \sum_{T \in \mathcal{T}_{H}} \eta_{T}^{2}
$$

holds. That is, triangles that carry most of the error were selected. The marked triangles were then refined with the red-green-refinement algorithm, which results in a finer mesh $\mathcal{T}_{h}$. In the error indicator $\eta$ given by Theorem 6.2, e.g. (6.5), we used $\left(y_{h}, u_{h}, p_{h}, \lambda_{h}\right)=\left(y_{\alpha, H}, u_{\alpha, H}, p_{\alpha, H}, \lambda_{\alpha, H}\right)$, i.e. the approximation of the discrete solution were taken as test functions.

The problem data is chosen according to Example 7.3, where we set $\alpha=10^{-4}$ and $\beta=1.5 \times 10^{-4}$. The problem was discretized using P1-elements for states and adjoints and P0-elements for the control. Hence we can expect $\left\|u_{\alpha, h}-u_{\alpha}\right\|_{L^{2}} \sim h$ due to Propositions 4.5 and 4.6. As solution algorithm we used the semi-smooth Newton method, see Stadler [27].

Starting with a mesh with 128 triangles, we computed a sequence of solutions and adaptively generated meshes. For comparison we computed solutions of the discretized problem for uniform refined meshes.

In Figure 7, we plotted the $L^{2}$-norms of the error $u_{\alpha, h}-u_{\alpha}$ and the values of the error estimator, i.e. $e_{u}:=\left\|u_{\alpha, h}-u_{\alpha}\right\|_{L^{2}}$ and $\eta_{u}:=\rho \sqrt{\sum \eta_{T}^{2}}$. The scaling factor $\rho$ was chosen such that the scaled error estimator coincides with the true error on the coarsest mesh. As one can expect, the adaptive process yields better approximation results: with the same number of unknowns the error is significantly smaller than for uniform 


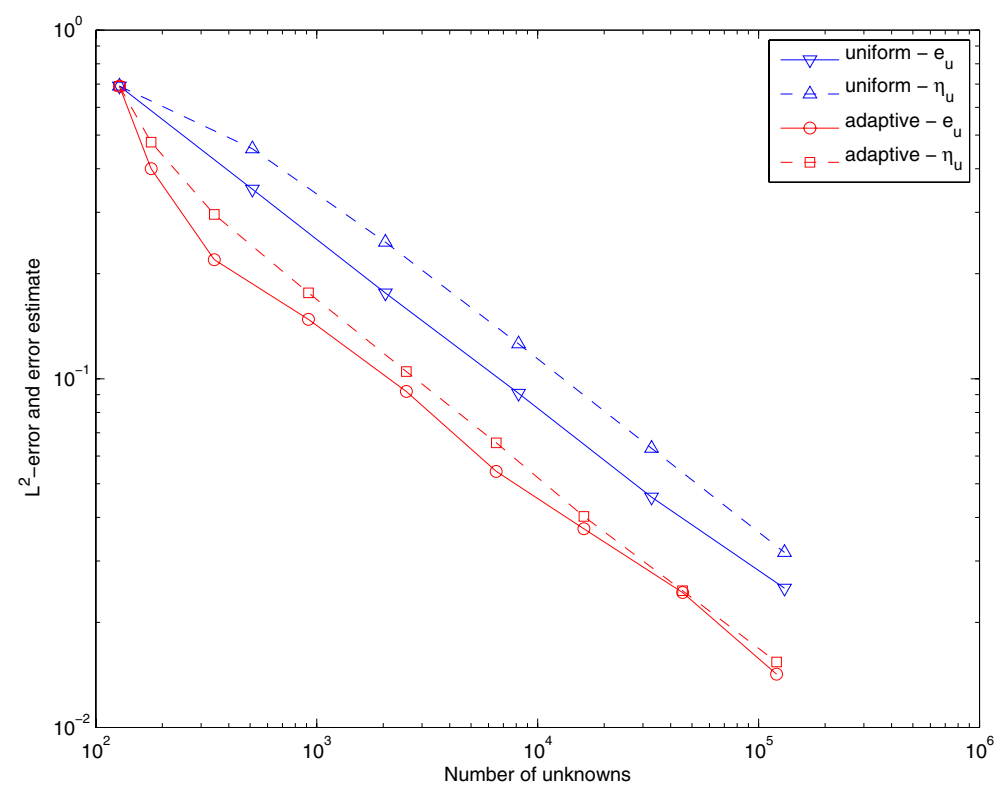

Figure 7. Errors in the control $e_{u}$ and scaled error estimator $\eta_{u}$.

refined meshes. Moreover, the plot of the error estimator shows that estimator and error are of the same order. This indicates that the error estimator is both reliable and efficient.

\section{A. Proof of Lemma 3.5}

At first let us state the following convergence result, which is cited from [30], Lemma 2.24, without proof.

Lemma A.1. Let $f(h) \in L^{2}(\Omega)$ for all $h \in U_{\delta}(0)$ with $\delta>0$ and let $f(h) \rightarrow \bar{f}$ in $L^{2}(\Omega)$ for $h \searrow 0$, i.e.,

$$
\lim _{h \searrow 0}\|f(h)-\bar{f}\|_{L^{2}} \rightarrow 0
$$

Then for $g \in L^{2}(\Omega)$, with $g(x)>0$ we have

$$
\begin{aligned}
\max \left(-\frac{g}{h}, f(h)\right) & \rightarrow \bar{f} & & \text { as } h \searrow 0, \\
\max (0, f(h)) & \rightarrow \max (0, \bar{f}) & & \text { as } h \searrow 0, \\
\max \left(0, f(h)-\frac{g}{h}\right) & \rightarrow 0 & & \text { as } h \searrow 0
\end{aligned}
$$

Now we are able to proof the differentiability of $\left(u_{\alpha}, y_{\alpha}, p_{\alpha}\right)$.

Proof of Lemma 3.5. The idea of the proof can be found in [27], proof of (3.11).

In a first step, we show that the difference quotient of $u_{\alpha}$ converges in the weak topology for almost all $\alpha>0$. After this, we obtain that this limit exists also in the strong topology and thus is the Gâteaux-derivative of $u_{\alpha}$.

As can be seen from Lemma 3.1, the mapping $\alpha \mapsto u_{\alpha}$ is locally Lipschitz continuous from $(0,+\infty)$ to $L^{2}(\Omega)$. Moreover, for every $\varepsilon>0$, the mapping $\alpha \mapsto u_{\alpha}$ is globally Lipschitz continuous from $[\varepsilon,+\infty)$ to $L^{2}(\Omega)$. Let $\varepsilon>0$ be arbitrary. Then it is well-known, see e.g. Aronszajn [1], p. 165, that $\alpha \mapsto u_{\alpha}$ is weakly differentiable 
almost everywhere on $[\varepsilon,+\infty)$. Therefore, we have for almost all $\alpha>\varepsilon$

$$
\frac{1}{\alpha-\alpha^{\prime}}\left(u_{\alpha}-u_{\alpha^{\prime}}\right) \rightarrow \dot{u}_{\alpha} \text { in } L^{2}(\Omega) \text { as } \alpha^{\prime} \rightarrow \alpha
$$

Since $\varepsilon>0$ was arbitrary, this convergence result holds for almost all $\alpha>0$.

Let us recall that operator $\mathcal{S}: L^{2}(\Omega) \rightarrow L^{2}(\Omega)$ is compact, while $\mathcal{S}^{*}: L^{2}(\Omega) \rightarrow L^{\infty}(\Omega)$ is continuous, which implies strong convergence of the difference quotients for $y_{\alpha}$ and $p_{\alpha}$

$$
\begin{gathered}
\frac{y_{\alpha^{\prime}}-y_{\alpha}}{\alpha^{\prime}-\alpha}=\frac{\mathcal{S}\left(u_{\alpha^{\prime}}-u_{\alpha}\right)}{\alpha^{\prime}-\alpha} \rightarrow \mathcal{S} \dot{u}_{\alpha}=: \dot{y}_{\alpha} \quad \text { in } L^{2}(\Omega) \text { as } \alpha^{\prime} \rightarrow \alpha, \\
\frac{p_{\alpha^{\prime}}-p_{\alpha}}{\alpha^{\prime}-\alpha}=\frac{-\mathcal{S}^{*}\left(y_{\alpha^{\prime}}-y_{\alpha}\right)}{\alpha^{\prime}-\alpha} \rightarrow-\mathcal{S}^{*} \dot{y}_{\alpha}=: \dot{p}_{\alpha} \quad \text { in } L^{\infty}(\Omega) \text { as } \alpha^{\prime} \rightarrow \alpha .
\end{gathered}
$$

For the remainder of the proof, let us choose $\alpha>0$, such that $\alpha \mapsto u_{\alpha}$ is (weakly) differentiable at $\alpha$. Let us write $u_{\alpha}$ as (cf. Fig. 1 and [27])

$$
\begin{aligned}
u_{\alpha}= & \alpha^{-1}\left(\max \left(0, p_{\alpha}-\beta\right)+\min \left(0, p_{\alpha}+\beta\right)\right. \\
& \left.-\max \left(0, p_{\alpha}-\beta-\alpha u_{b}\right)-\min \left(0, p_{\alpha}+\beta-\alpha u_{a}\right)\right) .
\end{aligned}
$$

Let us compute the directional derivatives $\dot{u}_{\alpha}^{+}, \dot{u}_{\alpha}^{-}$of the right hand side of (A.4) with respect to $\alpha$ in the directions +1 and -1 . We will show that both associated difference quotients converge strongly in $L^{2}(\Omega)$. By the weak convergence of the difference quotient of $u_{\alpha}$, we obtain $\dot{u}_{\alpha}=\dot{u}_{\alpha}^{+}=-\dot{u}_{\alpha}^{-}$and thus the strong convergence of the difference quotient.

Let us exemplarily calculate the convergence of the derivative in direction +1 of the first addend of (A.4). The arguments for the other terms of (A.4) and the derivative in direction -1 are analogous.

Let us define $g(\alpha):=\frac{p_{\alpha}-\beta}{\alpha}$ and $f(h):=\frac{1}{h}(g(\alpha+h)-g(\alpha))$. Then we have

$$
\lim _{h \searrow 0} f(h)=\dot{g}(\alpha)=\frac{\dot{p}_{\alpha}}{\alpha}+\frac{\beta-p_{\alpha}}{\alpha^{2}} .
$$

Now we show the convergence of the difference quotient of $\max (0, g(\alpha))$, i.e. the strong convergence of $\frac{1}{h}(\max (0, g(\alpha+h))-\max (0, g(\alpha)))$. We analyze the behavior of the difference quotient on different subsets of $\Omega$.

Case 1. On the set $G_{1}:=\{x \in \Omega: g(\alpha)(x)>0\}$ it holds

$$
\begin{aligned}
\frac{1}{h}(\max (0, g(\alpha+h))-\max (0, g(\alpha))) & =\frac{1}{h}[\max (0, g(\alpha+h))-g(\alpha)] \\
& =\max \left(-\frac{g(\alpha)}{h}, f(h)\right) \rightarrow \dot{g}(\alpha) \text { in } L^{2}\left(G_{1}\right) \text { as } h \searrow 0,
\end{aligned}
$$

where we refer to (A.1) in Lemma A.1.

Case 2. On the set $G_{2}:=\{x \in \Omega: g(\alpha)(x)=0\}$, we find using (A.2) in Lemma A.1

$$
\begin{aligned}
\frac{1}{h}(\max (0, g(\alpha+h))-\max (0, g(\alpha))) & =\frac{1}{h}[\max (0, g(\alpha+h))] \\
& =\max (0, f(h)) \rightarrow \max (0, \dot{g}(\alpha)) \text { in } L^{2}\left(G_{2}\right) \text { as } h \searrow 0 .
\end{aligned}
$$


Case 3. On the set $G_{3}:=\{x \in \Omega: g(\alpha)(x)<0\}$, we obtain

$$
\begin{aligned}
\frac{1}{h}(\max (0, g(\alpha+h))-\max (0, g(\alpha))) & =\frac{1}{h}[\max (0, g(\alpha+h))] \\
& =\max \left(0, f(h)+\frac{g(\alpha)}{h}\right) \rightarrow 0 \text { in } L^{2}\left(G_{3}\right) \text { as } h \searrow 0,
\end{aligned}
$$

where we applied (A.3) of Lemma A.1.

Thus, the directional derivative of $\max (0, g(\alpha))$ in direction 1 is given by

$$
\dot{g}(\alpha) \chi_{I},
$$

where $I=\{x \in \Omega: g(\alpha)(x)>0$ or $g(\alpha)(x)=0, \dot{g}(\alpha)(x) \geq 0\}$. Moreover, due to (A.5)-(A.7), the difference quotients of $g(\alpha)$ converge strongly to $\dot{g}(\alpha)$ in $L^{2}(\Omega)$.

The calculations for the other addends are similar and yield strong convergence of the difference quotients. Defining

$$
\begin{aligned}
& I_{1}:=\left\{p_{\alpha} \in\left(\beta, \beta+\alpha u_{b}\right) \text { or } p_{\alpha}=\beta, \dot{p}_{\alpha} \geq 0 \text { or } p_{\alpha}=\beta+\alpha u_{b}, \dot{p}_{\alpha} \leq \alpha u_{b}\right\} \\
& I_{2}:=\left\{p_{\alpha} \in\left(-\beta+\alpha u_{a},-\beta\right) \text { or } p_{\alpha}=-\beta, \dot{p}_{\alpha} \leq 0 \text { or } p_{\alpha}=-\beta+\alpha u_{a}, \dot{p}_{\alpha} \geq \alpha u_{a}\right\}
\end{aligned}
$$

we obtain

$$
\dot{u}_{\alpha}^{+}=\left(-\frac{1}{\alpha^{2}} p_{\alpha}+\frac{1}{\alpha} \dot{p}_{\alpha}\right) \chi_{I_{1} \cup I_{2}}+\frac{\beta}{\alpha^{2}}\left(\chi_{I_{1}}-\chi_{I_{2}}\right) .
$$

The calculation of the directional derivative in direction -1 gives with

$$
\begin{aligned}
& I_{3}:=\left\{p_{\alpha} \in\left(\beta, \beta+\alpha u_{b}\right) \text { or } p_{\alpha}=\beta, \dot{p}_{\alpha} \leq 0 \text { or } p_{\alpha}=\beta+\alpha u_{b}, \dot{p}_{\alpha} \geq \alpha u_{b}\right\} \\
& I_{4}:=\left\{p_{\alpha} \in\left(-\beta+\alpha u_{a},-\beta\right) \text { or } p_{\alpha}=-\beta, \dot{p}_{\alpha} \geq 0 \text { or } p_{\alpha}=-\beta+\alpha u_{a}, \dot{p}_{\alpha} \leq \alpha u_{a}\right\}
\end{aligned}
$$

the expression

$$
-\dot{u}_{\alpha}^{-}=\left(-\frac{1}{\alpha^{2}} p_{\alpha}+\frac{1}{\alpha} \dot{p}_{\alpha}\right) \chi_{I_{3} \cup I_{4}}+\frac{\beta}{\alpha^{2}}\left(\chi_{I_{3}}-\chi_{I_{4}}\right) .
$$

Let us compare equations (A.8) and (A.9) for $\dot{u}_{\alpha}$. Multiplying these equations with the characteristic functions of the sets $\left\{p_{\alpha}=\beta\right\},\left\{p_{\alpha}=\beta+\alpha u_{b}\right\},\left\{p_{\alpha}=-\beta\right\}$ and $\left\{p_{\alpha}=-\beta+\alpha u_{a}\right\}$, we obtain

$$
\begin{array}{ll}
p_{\alpha}=\beta & \Rightarrow \dot{p}_{\alpha}=0 \\
p_{\alpha}=\beta+\alpha u_{b} & \Rightarrow \dot{p}_{\alpha}=\alpha u_{b} \\
p_{\alpha}=-\beta & \Rightarrow \dot{p}_{\alpha}=0 \\
p_{\alpha}=-\beta+\alpha u_{a} & \Rightarrow \dot{p}_{\alpha}=\alpha u_{a} .
\end{array}
$$

Hence (A.9) implies that the derivative of $u_{\alpha}$ on these sets is 0 and

$$
\dot{u}_{\alpha}=\left(-\frac{1}{\alpha^{2}} p_{\alpha}+\frac{1}{\alpha} \dot{p}_{\alpha}\right) \chi_{I_{1} \cup I_{2}}+\frac{\beta}{\alpha^{2}}\left(\chi_{I_{1}}-\chi_{I_{2}}\right)
$$

holds, where

$$
\begin{aligned}
& I_{1}=\left\{p_{\alpha} \in\left(\beta, \beta+\alpha u_{b}\right)\right\} \\
& I_{2}=\left\{p_{\alpha} \in\left(-\beta+\alpha u_{a},-\beta\right)\right\} .
\end{aligned}
$$


Therefore the sets $I_{1}$ and $I_{2}$ are independent of $\dot{p}_{\alpha}$. Together with the equations

$$
\begin{aligned}
& \dot{y}_{\alpha}=\mathcal{S} \dot{u}_{\alpha}, \\
& \dot{p}_{\alpha}=-\mathcal{S}^{\star} \dot{y}_{\alpha}
\end{aligned}
$$

we obtain the linear system for the derivative $\left(\dot{u}_{\alpha}, \dot{y}_{\alpha}, \dot{p}_{\alpha}\right)$ as claimed.

Acknowledgements. The authors wish to thank Anton Schiela for intensive discussions that led to the result of Theorem 3.7, and improved the convergence rates in the preprint version of this article.

\section{REFERENCES}

[1] N. Aronszajn, Differentiability of Lipschitzian mappings between Banach spaces. Studia Math. 57 (1976) 147-190.

[2] R. Becker and R. Rannacher, An optimal control approach to a posteriori error estimation in finite element methods. Acta Numer. 10 (2001) 1-102.

[3] C. Carstensen, Quasi-interpolation and a posteriori error analysis in finite element methods. ESAIM: M2AN 33 (1999) 11871202.

[4] E. Casas and M. Mateos, Error estimates for the numerical approximation of boundary semilinear elliptic control problems. Continuous piecewise linear approximations, in Systems, control, modeling and optimization 202, IFIP Int. Fed. Inf. Process., Springer, New York (2006) 91-101.

[5] C. Clason and K. Kunisch, A duality-based approach to elliptic control problems in non-reflexive Banach spaces. ESAIM: COCV (2010) DOI: $10.1051 / \mathrm{cocv} / 2010003$.

[6] I. Daubechies, M. Defrise and C. De Mol, An iterative thresholding algorithm for linear inverse problems with a sparsity constraint. Comm. Pure Appl. Math. 57 (2004) 1413-1457.

[7] D. Donoho, For most large underdetermined systems of linear equations the minimal $l^{1}$-norm solution is also the sparsest solution. Comm. Pure Appl. Math. 59 (2006) 797-829.

[8] A.L. Dontchev, W.W. Hager, A.B. Poore and B. Yang, Optimality, stability and convergence in optimal control. Appl. Math. Optim. 31 (1995) 297-326.

[9] R.S. Falk, Approximation of a class of optimal control problems with order of convergence estimates. J. Math. Anal. Appl. 44 (1973) 28-47.

[10] M. Grasmair, M. Haltmeier and O. Scherzer, Sparse regularization with $l^{q}$ penalty term. Inv. Prob. 24 (2008) 055020.

[11] R. Griesse and D.A. Lorenz, A semismooth Newton method for Tikhonov functionals with sparsity constraints. Inv. Prob. 24 (2008) 035007.

[12] R. Griesse, T. Grund and D. Wachsmuth, Update strategies for perturbed nonsmooth equations. Optim. Methods Softw. 23 (2008) 321-343.

[13] M. Hintermüller, R.H.W. Hoppe, Y. Iliash and M. Kieweg, An a posteriori error analysis of adaptive finite element methods for distributed elliptic control problems with control constraints. ESAIM: COCV 14 (2008) 540-560.

[14] M. Hinze, A variational discretization concept in control constrained optimization: the linear-quadratic case. Comp. Optim. Appl. 30 (2005) 45-63.

[15] A.D. Ioffe and V.M. Tichomirov, Theorie der Extremalaufgaben. VEB Deutscher Verlag der Wissenschaften, Berlin (1979).

[16] B. Jin, D.A. Lorenz and S. Schiffler, Elastic-net regularization: error estimates and active set methods. Inv. Prob. 25 (2009) 115022 .

[17] K. Krumbiegel and A. Rösch, A new stopping criterion for iterative solvers for control constrained optimal control problems. Archives of Control Sciences 18 (2008) 17-42.

[18] R. Li, W. Liu, H. Ma and T. Tang, Adaptive finite element approximation for distributed elliptic optimal control problems. SIAM J. Control Optim. 41 (2002) 1321-1349.

[19] R. Li, W. Liu and N. Yan, A posteriori error estimates of recovery type for distributed convex optimal control problems. J. Sci. Comput. 33 (2007) 155-182.

[20] W. Liu and N. Yan, A posteriori error estimates for convex boundary control problems. SIAM J. Numer. Anal. 39 (2001) 73-99.

[21] D.A. Lorenz, Convergence rates and source conditions for Tikhonov regularization with sparsity constraints. J. Inverse Ill-Posed Probl. 16 (2008) 463-478.

[22] D.A. Lorenz and A. Rösch, Error estimates for joint Tikhonov- and Lavrentiev-regularization of constrained control problems. Appl. Anal. (to appear).

[23] C. Meyer and A. Rösch, Superconvergence properties of optimal control problems. SIAM J. Control Optim. 43 (2004) $970-985$.

[24] C. Meyer, J.C. de los Reyes and B. Vexler, Finite element error analysis for state-constrained optimal control of the Stokes equations. Control Cybern. 37 (2008) 251-284. 
[25] R. Ramlau and G. Teschke, A Tikhonov-based projection iteration for nonlinear ill-posed problems with sparsity constraints. Numer. Math. 104 (2006) 177-203.

[26] A. Schiela, Barrier methods for optimal control problems with state constraints. SIAM J. Optim. 20 (2009) $1002-1031$.

[27] G. Stadler, Elliptic optimal control problems with $L^{1}$-control cost and applications for the placement of control devices. Comp. Optim. Appl. 44 (2009) 159-181.

[28] G. Stampacchia, Le problème de Dirichlet pour les équations elliptiques du second ordre à coefficients discontinus. Ann. Inst. Fourier (Grenoble) 15 (1965) 189-258.

[29] F. Tröltzsch, Optimale Steuerung partieller Differentialgleichungen. Vieweg, Wiesbaden (2005).

[30] G. Wachsmuth, Elliptische Optimalsteuerungsprobleme unter Sparsity-Constraints. Diploma Thesis, Technische Universität Chemnitz (2008) http://www.tu-chemnitz.de/mathematik/part_dgl/publications.php. 